INRA Prod. Anim., 2017, 30 (2), 179-196

\section{Étude prospective des filières viande de ruminants du Massif central à l'horizon 2050}

I INRA, UMR 1213 herbivores, Theix, 63122 Saint-Genès-Champanelle, France 2 ASCA, 8 rue Legouvé, 75010 Paris, France

Courriel : jacques.agabriel@inra.fr

Les filières viande du Massif central partagent beaucoup d'inquiétudes sur leur avenir. Pour se donner les moyens d'anticiper 2050, le Commissariat Général à l'Égalité des Territoires a initié une étude prospective qui a produit cinq scénarios contrastés de futurs possibles. Leurs conséquences économiques, environnementales et sociales sont analysées.

Le Massif central est considéré en France comme un grand territoire d'élevage, berceau de races bovines et ovines et pilier d'une forte activité plutôt extensive. A titre d'illustration, sa charge ou pression animale moyenne tous animaux confondus pour les anciennes régions d'Auvergne et du Limousin n'est que de 1,04 UGB/ ha SAU (RICA 2014), en contraste fort avec l'estimation de 2,58 UGB /ha SAU du grand ouest (régions Bretagne et Pays de Loire). Une étude conduite par le Service InterDépartemental pour l'Animation du Massif central (SIDAM) et l'Institut de l'Élevage (Idele) avait fait l'état des lieux des filières en 2013 (Sanne et al 2013), et déjà souligné la forte spécialisation des exploitations du Massif central vers la production de viande bovine (environ un tiers contre $12 \%$ pour l'ensemble du pays). Mais les alternatives sont limitées dans un territoire où $96 \%$ des exploitations agricoles se situent en zone de montagne $(58 \%)$ ou de piémont $(16 \%)$ ou défavorisées simples $(17 \%)$ et où la prairie permanente (Surface Toujours en Herbe, STH) recouvre $60 \%$ de la Surface Agricole Utile (SAU).

Déjà déstabilisés par la récurrence de faibles revenus agricoles (Veysset et al 2014), les élevages du Massif se trouvent fragilisés par les transformations sociales, économiques et sociétales, par la faible attractivité des métiers de l'élevage, et par les risques climatiques qui pèsent sur le fonctionnement des systèmes fourragers des exploitations.

Mais au-delà des exploitations, c'est toutes les filières qui sont fragilisées par la baisse régulière de la consommation individuelle des viandes de ruminants en Europe (Crédoc 2012). Rares sont les marchés de substitution et une spirale dégressive pourrait s'installer entre la baisse de la demande et une baisse des prix. De plus, l'évolution des modes de consommation de viande rouge se traduit par une demande et une place de plus en plus importante du marché de la viande bovine hachée qui ne valorise que partiellement les carcasses lourdes et bien conformées des races du Massif.

Ces évolutions touchent les grands pays européens de façon similaire et notamment l'Italie dont le marché est le débouché principal des bovins mâles maigres produit dans le Massif central (cf. Lherm et al 2017, ce numéro). La fragilisation de ce débouché pèse ainsi fortement sur l'avenir des élevages naisseurs du Charolais ou du sud Aubrac au regard de leur organisation actuelle. La durabilité économique de ces filières spécialisées est ici clairement interrogée. Elle repose également sur la structure des exploitations familiales dont la capacité de transmission générationnelle reste forte mais diminue. Les passations se faisaient jusque-là dans un même monde professionnel quitte à regrouper les plus petites exploitations pour viser des économies d'échelles et des gains de productivité du travail. Mis en évidence, et décrits dans les suivis de fermes sur le temps long, on en aperçoit désormais les limites (Veysset et al 2014). Alors que l'âge moyen des éleveurs du Massif augmente, le problème de transmission des exploitations s'accentue et se trouve décuplé par la double augmentation de la taille et du capital investi.

Le Massif central se retrouve ainsi au cœur des débats sociétaux actuels qui interrogent l'avenir de l'activité d'élevage de ruminants. Ces discussions inquiètent d'autant plus les producteurs car elles touchent aux fondements même de leurs métiers. Ils se voient ainsi interpellés alors qu'ils ont le sentiment de " bien faire » dans le cadre de systèmes d'élevage qu'ils considèrent comme " naturels » et « à taille humaine ».

Évoluer donc, mais comment? Que remettre en cause ou au contraire conforter par des investissements collectifs ? Les trois pistes ouvertes dans le rapport SIDAM-Idèle proposaient soit la relance de l'engraissement et de la production, soit à l'opposé son déclin et le boisement des surfaces libérées, soit enfin la continuation de la tendance actuelle d'agrandissement des structures et l'extensification des troupeaux. Très focalisés sur la production de bovins allaitants et sur le court et moyen termes, ces petites pistes ne suffisaient pas pour réinterroger les champs des futurs possibles. Ainsi pour aider les acteurs des filières d'élevage de ruminants et les usagers de son territoire à affronter ces évolutions, le Commissariat Général à l'Égalité des Territoires (CGET) a chargé le centre INRA Auvergne-Rhône-Alpes (ARA) de la réalisation d'une étude prospective concernant $l$ 'avenir des filières viande de ruminants du Massif central à l'horizon $2050^{1}$. La commande englobait tous les stades des filières, c'est-à-dire la

${ }^{1}$ Étude disponible à l'adresse : http://www.ara.inra.fr rubrique Le-centre-Les-recherches/projets-et-actualités. 
Figure 1. Présentation du territoire d'étude : le Massif central et ses principaux bassins d'élevage.

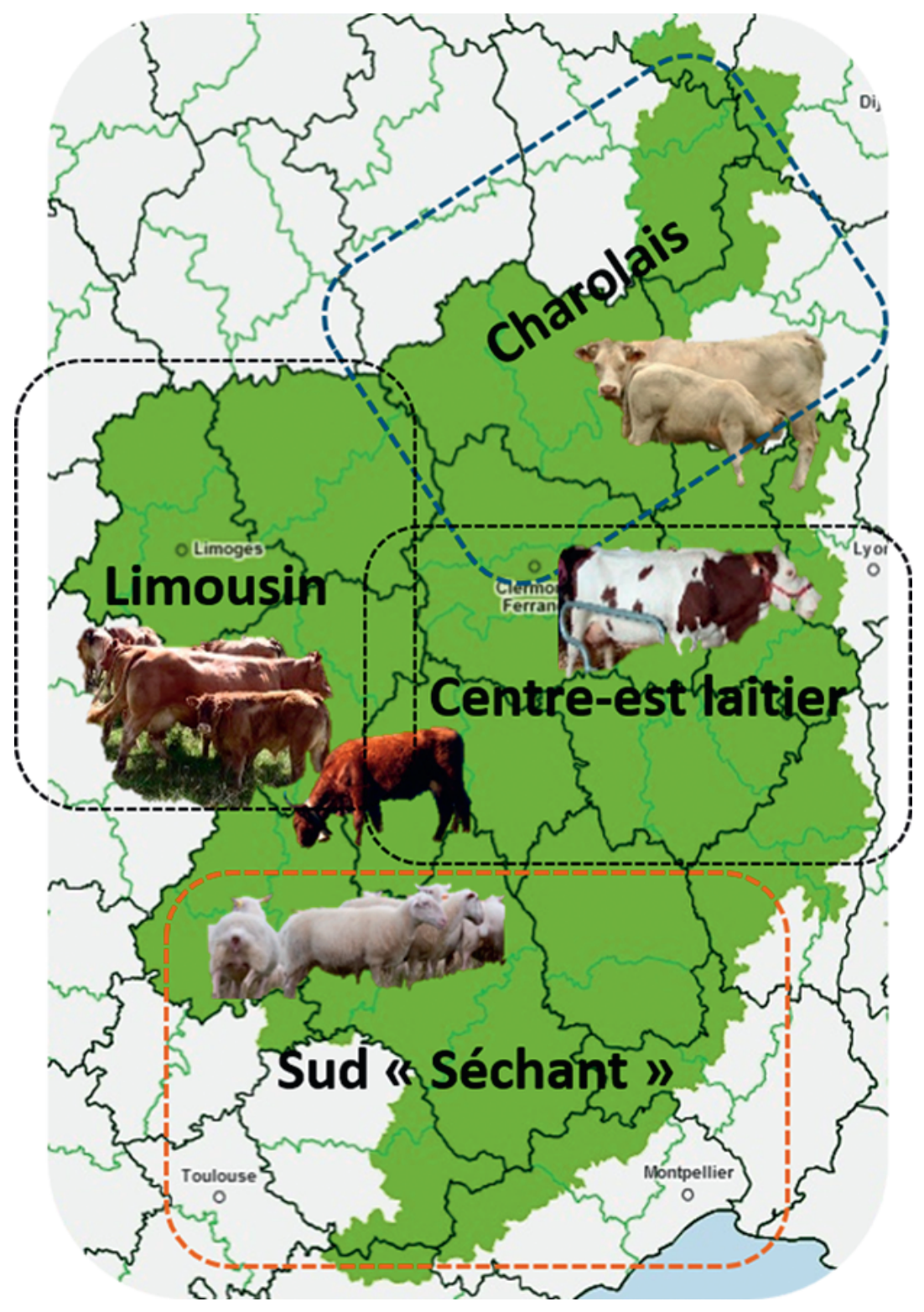

production, la transformation, la commercialisation et la consommation. Elle intégrait les productions marchandes issues des filières allaitantes et laitières (animaux, viande) et les productions non marchandes (services rendus par cette agriculture). Au-delà d'une meilleure connaissance du « système Massif central », les multiples attentes des commanditaires par rapport à ce travail étaient clairement identifiées :

- Faire ressortir les marges de manœuvre et d'adaptation dans des contextes futurs contrastés. La projection dans le futur force à anticiper ce qu'il faudrait faire, si celui-ci se réalisait. On trouve ici une dimension stratégique pour à la fois élargir la gamme des actions et en évaluer les tenants et aboutissants dans différents contextes. Cette approche invite à remettre en cause la proposition de solutions uniques.
- Proposer un outil d'aide à la décision stratégique aux porteurs d'enjeux, qu'ils soient acteurs des filières (production, transformation, distribution) ou des territoires (acteurs de l'environnement et de l'aménagement).

- Constituer un groupe de réflexion et de discussion partageant des connaissances sur l'objet d'étude et faire émerger des innovations et des pistes de recherche parmi les conditions de réussite des scénarios élaborés. À l'aune de ce travail, l'objectif est que l'ensemble des professionnels de la chaîne de production de viande de ruminants puissent prendre des décisions susceptibles d'entretenir une activité économique renouvelée synonyme d'attractivité pour le territoire.

In fine, cette étude invite les porteurs d'enjeux du Massif central à se saisir dès maintenant de ces questions priori- taires et d'accompagner au mieux les filières dans leurs mutations.

L'objectif de cet article est de faire une synthèse de cette étude prospective, qui au-delà de la description factuelle des cinq scénarios, mette en avant les originalités méthodologiques de leur élaboration. De plus, les quantifications des impacts associés aux hypothèses, en termes d'utilisation du territoire, de production, d'emploi et d'environnement sont également analysées et discutées pour mieux comparer les différences que ces divers futurs peuvent amener.

\section{1 / État des filières de rumi- nants du Massif central}

\section{1 / En 2010 une place centrale en France voire même en Europe}

Composé de tout ou partie de 22 départements essentiellement de moyenne montagne, le Massif central (MC) (figure 1) représente $15 \%$ du territoire français sur lequel s'élèvent respectivement 35 et $34 \%$ des vaches et brebis nourrices françaises, et 12 et $55 \%$ des vaches et brebis laitières (tableau 1). L'entité administrative du Massif central n'est pas toujours considérée dans les statistiques nationales, et en raison du manque de données disponibles à l'échelle infra départementales (en particulier pour les séries anciennes), nous avons été amenés à retenir également un périmètre constitué de la totalité de seulement 18 départements (MC18). Il englobe les 11 départements entièrement dans le Massif (Les 4 départements de l'Auvergne, les 3 du Limousin, l'Aveyron, le Lot, la Lozère, la Loire) plus les 4 départements de la région Bourgogne, plus 1'Ardèche, le Tarn et le Rhône. C'est ce périmètre qui avait été retenu dans l'étude SIDAM/Idele (Sanne et al 2013), et dans cet article les références utilisant MC ou MC18 sont signalées si nécessaire.

La prairie, permanente ou temporaire, couvre plus de $80 \%$ de la SAU du Massif et structure les paysages (Agreste 2010 et 2015). Les filières de ruminants s'appuient donc en priorité sur cette ressource en herbe abondante et la valorise en produits de qualité. Le cheptel bovin laitier du MC18 est composé de près de 0,5 million de vaches et produit 2,2 milliards de litres de lait par an. Les 2/3 sont transformés sur le territoire, principalement en fromages avec $22 \%$ en filière $\mathrm{AOP}$ et $35 \%$ en autres fromages (Idèle 2010). Le cheptel ovin laitier a un statut particulier du fait de l'importance de la zone de Roquefort dans le sud du Massif (0,7 million de mères, $45 \%$ du fromage de brebis produit). Les vaches allaitantes représentent 1,8 million de mères qui se 
Tableau 1. Principaux indicateurs descriptifs du territoire du Massif central et en proportion par rapport à la France.

\begin{tabular}{|c|c|c|}
\hline & Massif central ${ }^{(1)}$ & $\begin{array}{c}\text { Part du Massif central } \\
\text { / France }(\%)\end{array}$ \\
\hline Surface totale (ha) & 8500000 & 15 \\
\hline SAU (ha) & 4130000 & 15 \\
\hline Emploi (Nombre d’UTA) & 98000 & 12 \\
\hline SAU Ruminants (ha) & 3400000 & 31 \\
\hline Exploitations Agricoles ruminants (Nombre) & 50500 & 28 \\
\hline Surface médiane exploitations agricoles (ha) & 60 & \\
\hline Surface toujours en herbe (ha) & 2460000 & 32 \\
\hline Viande bovine produite (ktec) & 300 & 20 \\
\hline Parcs régionaux / nationaux (Nombre) & $9 / 1$ & \\
\hline Revenus agricoles & Auvergne + Limousin ${ }^{(3)}$ & France \\
\hline $\mathrm{RCAI}^{(2)} / €$ UTAns Toutes exploitations & 19910 & 28620 \\
\hline $\mathrm{RCAI}^{(2)} / €$ UTAns OTEX 46 Bovin Viande & 19940 & 19320 \\
\hline
\end{tabular}

\footnotetext{
SAU = Surface Agricole Utile $;$ UTA = Unité de Travail Annuel ; RCAI = Revenu Courant Avant Impôts ; UTAns = Unité de Travail Annuel non salarié.

(1) Massif central périmètre CGET, Statistiques : Agreste RA 2010 ; ${ }^{(2)}$ Moyenne 2012-2014 source RICA ;

(3) Uniquement Auvergne + Limousin du fait de la non disponibilité des données pour le Massif central.
}

répartissent dans les trois grands bassins Charolais, Limousin et Montagne Sud. Elles forment $15 \%$ du total européen. Elles produisent chaque année 1,5 million d'animaux maigres dont 0,9 million de broutards mâles et femelles sevrés de 9 mois environ, qui sont ensuite vendus pour être engraissés en dehors du Massif (mais pour $42 \%$ en France). Le cheptel ovin allaitant (1,8 million de mères) produit majoritairement des agneaux de bergerie pour un marché français déficitaire en viande ovine. Les productions de viande du Massif trouvent des valorisations particulières dans les signes officiels de qualité puisqu'on en recense 13 pour les bovins ( 2 AOP, 6 IGP, 6 labels rouge) et 14 pour les ovins (7 IGP et 6 labels). Mais cette diversité a son revers puisque chacun regroupe peu de tonnages ce qui limite d'autant leurs propres renommées.

Au total plus de 90000 exploitations agricoles, dont plus de $60 \%$ ont des activités d'élevage, valorisent le territoire (MC18) et représenteraient 7\% des emplois totaux. Les autres emplois associés - près de 45000 - sont dans les secteurs de la collecte des produits, de leur transformation et de leur distribution ainsi que dans les services liés à l'élevage (Rieutort et al 2014, Ryschawy et al 2015, Dumont et al 2016). Les revenus de ces exploitations sont inférieurs de $30 \%$ (Auvergne et Limousin) au revenu moyen des exploitations françaises, mais essentiellement du fait des productions qui y sont menées. Ainsi dans une comparaison stricte des exploitations Bovins Élevage et Viande (Otex 46) les moyennes pour les régions Auvergne et Limousin et pour la France entière sont identiques (tableau 1).

Du fait de l'importance croissante de la filière de bovins maigres, une soixantaine d'abattoirs seulement demeurent dans le Massif et ne produisent que $20 \%$ de la production bovine nationale (environ $300 \mathrm{ktec}$ sur $1600 \mathrm{ktec}$ ) et $50 \mathrm{ktec}$ soit $60 \%$ de la production ovine.

\section{2 / Une situation actuelle issue des évolutions passées entre 1950 et 2010. Les éléments de rupture}

En retraçant l'évolution de l'appareil productif, des structures d'exploitation, du territoire et du paysage du Massif central depuis les années 1950, nous avons tout d'abord cherché à prendre le recul nécessaire à l'exercice de prospective. Le choix d'un temps long, en symétrique de la vision future à 35 ans, a permis de mettre en évidence et de partager les ruptures et les évolutions lentes intervenues au fil des décennies, mais aussi de réfléchir aux tendances et aux signaux faibles pouvant sous-tendre les scénarios à construire. L'analyse a porté sur le niveau macro de la France et de l'Union européenne en se focalisant sur le contexte démographique, les politiques européennes et nationales et les grandes évolutions de filières. Elle a aussi porté sur le niveau du Massif central en se focalisant sur les structures d'exploitation et l'utilisation du territoire avec ses conséquences paysagères. Cette histoire passée a été schématisée sous la forme d'une frise historique (figure 2) résumant les évolutions des dimensions analysées. Les grandes dynamiques à l'œuvre émergent de quatre images du passé $(1950,1970$, 1990 et 2010) finement décrites et paraissent façonner la situation actuelle :

- La politique de boisement des moins bonnes terres et landes dans les années 1960 sous l'impulsion du Fond Forestier National a conduit à la forte réduction de la SAU (Dodane 2009). Mais cette construction d'une mosaïque forêt / prairie qui n'existait pas ou peu a conduit à la naissance progressive d'une valeur « socioéconomique » de paysages contrastés, typiques désormais du Massif central.

- La forte concentration de l'activité agricole sur le $M C$ a réduit de 450000 à 98000 le nombre des exploitations. Cette baisse s'est faite pourtant avec la même intensité qu'au niveau national ; le Massif central représente invariablement $20 \%$ du total des exploitations françaises.

- Le remplacement de l'élevage laitier mixte par les bovins spécialisés viande s'est accéléré après la mise en place des quotas. La spécialisation de la filière « bovins allaitants » vers la production d'animaux maigres type broutards s'est progressivement consolidée. Ce développement s'est accompagné d'une organisation de l'offre et la mise en place de partenariats commerciaux privilégiés notamment avec les ateliers d'engraissement italiens. La filière de bovins maigres apparait ainsi très structurée et ajustée pour approvisionner les ateliers d'engraissement de la plaine du Pô. Les 
Figure 2. Frise rétrospective illustrant les grandes évolutions agricoles entre 1950 et 2010 à partir de la description de 4 images statiques (1950, 1970, 1990, 2010) et des processus à l'œuvre entre ces années.

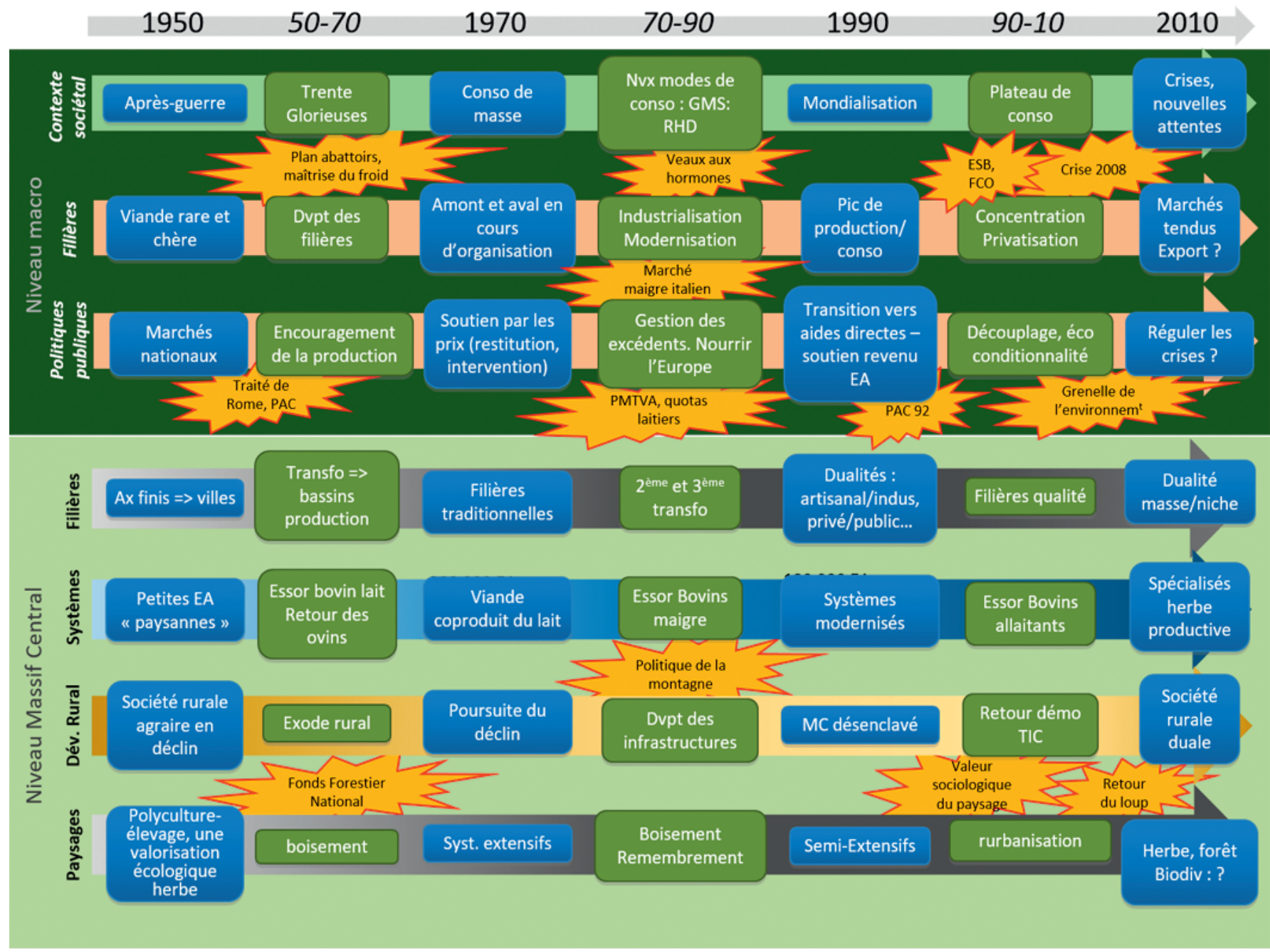

groupes français sont d'ailleurs présents dans le capital de sociétés de commerce et d'engraissement italiennes.

- L'encouragement public pour les productions et notamment les productions bovines allaitantes est continu et va croissant jusque vers 2000. Ainsi le soutien à la tête de vache (Prime au Maintien du Troupeau de Vaches Allaitantes - PMTVA puis Aides aux Bovins Allaitants - ABA) a été maintenu à la demande de la profession. Les aides directes issues de la Politique Agricole Commune (PAC) sont d'importance, et leur montant total dépasse le revenu des éleveurs allaitants depuis 1995. Le Massif bénéficie également de la mise en place d'une politique spécifique de soutien à l'agriculture de montagne et aux zones défavorisées dès 1975.

- Les crises aiguës et récurrentes sur la consommation ne modifient que transitoirement les habitudes des consommateurs : la baisse régulière de la consommation individuelle de viande n'intervient que depuis le début des années 1990. La crise de l'Encéphalopathie Spongiforme Bovine (ESB) est « la crise mère » qui a modifié le regard majoritaire. La viande rouge a depuis peu à peu perdu son image des années 1950, c'est-à-dire celle d'un produit rare, cher et indispensable à la santé. Sa consommation (par tête) est passée par un maximum en 1979 avant de se réduire tendanciellement de $0,8 \%$ par an.

- Enfin aiguillonnée par les crises, la filière s'est beaucoup transformée. Les outils d'abattage se sont concentrés et fortement industrialisés. Ils se sont éloignés des centres urbains. Mais à l'inverse les acteurs intermédiaires et de négoce se sont maintenus et restent bien présents. La mise en place de la traçabilité rapprochée des produits en réponse à une demande forte des consommateurs est maintenant quasiment aboutie. Le développement récent de la viande hachée l'a rendu encore plus nécessaire et a poussé à sa mise en place en routine jusqu'au niveau des muscles, avec le PAD (Prêt A Découper). Cette traçabilité est ainsi un processus de long terme : elle a de fait commencée avec l'identification généralisée des animaux dans les années 1960-70.

\section{2 / Méthodologie de la démar- che prospective}

Une dizaine de représentants des principaux acteurs du Massif (éleveurs, transformateurs, distributeurs, parcs régionaux et élus) ont été réunis en un comité de suivi du projet. Pour traiter de l'identification des enjeux, la démarche visait à croiser les thèmes (économie, zootechnie, développement local, environnement) et les niveaux d'expertise (local, Massif, national et supra national). Si cette identification relevait de la compétence du comité de suivi, les scénarios destinés à les éclairer sont issus d'un autre groupe de travail pluri-thématique composé d'une vingtaine de membres indépendants, associant des experts scientifiques et techniques externes au Massif et des acteurs locaux (filières, Parcs Naturels Régionaux - PNR, associations), euxmêmes en capacité d'expertise locale. Ces experts ont construit les scénarios au cours de cinq journées de travail collectif qui se sont étalées sur 14 mois. Des entretiens supplémentaires, ciblés 
Figure 3. Les familles d'enjeux identifiés par les acteurs du système Massif central. Certains enjeux peuvent appartenir à plusieurs familles.

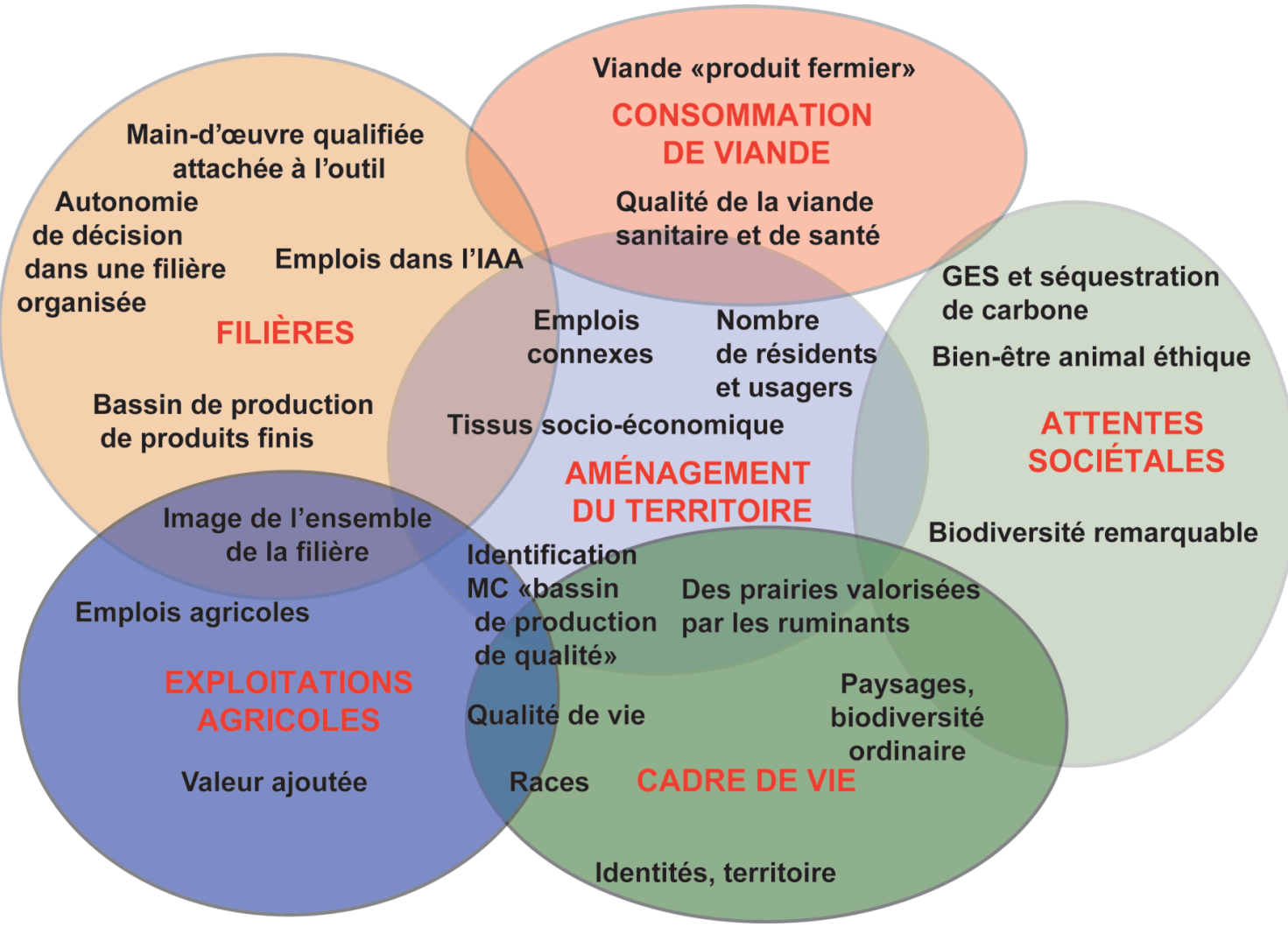

avec une trentaine de personnes ayant une compétence ou une activité spécifique, ont été menés pour compléter les informations, enrichir et élargir les points de vue.

\section{1 / Les étapes de la démarche}

\section{a) Identification des enjeux}

L'identification d'une liste d'enjeux a fait l'objet de la première session de travail du comité de suivi. Par enjeu on comprend ce que les différents types d'acteurs ont à gagner ou à perdre à l'horizon 2050, en liens avec les évolutions envisageables des filières. Un enjeu est situé : il se rapporte à une catégorie d'acteurs, qui peuvent être internes (par exemple les éleveurs du Massif qui auront, dans le futur, à " gagner » ou à « perdre » du revenu agricole) ou plus externes comme les touristes randonneurs (qui auront à « gagner » ou « perdre » des paysages attractifs). Les enjeux constituent ainsi les « variables à expliquer » par la prospective pour les différents acteurs de Massif : c'est essentiellement d'eux dont les scénarios devront rendre compte. À ce titre, ils organisent l'ensemble de la réflexion.

\section{b) Regroupement des enjeux}

Une fois cette liste réalisée, les enjeux ont été regroupés en méta-enjeux plus faciles à manipuler. Six grands groupes ont ainsi été mis en exergue et organisés dans une représentation morphologique partagée (figure 3) : i) les exploitations agricoles, $i$ i) les filières, iii) l'aménagement du territoire, $i v$ ) la consommation de viande, $v$ ) les attentes sociétales et $v i$ ) le cadre de vie.

\section{c) Déterminants influençant les enjeux}

Certaines variables peuvent influer sur l'évolution des enjeux. On peut par exemple citer le prix de l'énergie, le réchauffement climatique, etc. En partant d'une analyse systématique des enjeux, ces variables explicatives ont été répertoriées puis regroupées en grands ensembles - appelés « déterminants »modulant l'avenir du système d'enjeux. Le rôle de ces déterminants a été explicité par une analyse rétrospective depuis les années 1950, révélant les forces motrices expliquant l'évolution des différentes familles d'enjeux. Ces forces sont le fait de dynamiques externes au Massif central, sur lesquelles les acteurs des filières n'ont pas de prise et doivent s'adapter (par exemple l'évolution de la consommation de viande en Europe). D'autres sont davantage internes au système, et les acteurs peuvent plus facilement les mobiliser (par exemple l'organisation professionnelle). Les déterminants internes représentent ainsi de véritables leviers actionnables par les acteurs du territoire alors que les déterminants externes constituent tous ensemble le « décor» (ou contexte) dans lequel ces acteurs doivent intervenir.

d) Classification et analyse des déterminants, élaboration des décors et des scénarios

Six grands déterminants ont ainsi été identifiés et classés comme externes $(n=4)$ ou internes $(n=2)$. Pour chacun d'entre eux, il s'agissait d'imaginer des hypothèses plausibles d'évolution à l'horizon 2050 (pour chaque famille, une hypothèse future correspond à une « modalité »).

i) La consommation de viande à l'échelle européenne (déterminant externe 1 : 4 modalités différentes) ;

ii) l'organisation des grandes filières viande européennes (déterminant externe $2: 4$ modalités) ;

iii) les politiques agricoles et environnementales sectorielles et les accords internationaux (déterminant externe 3 : 4 modalités) ;

iv) Le changement climatique (déterminant externe 4) : une seule modalité selon les hypothèses du Groupe d'experts intergouvernemental sur l'évolution du climat (GIEC) à horizon proche ;

v) l'aménagement du territoire : démographie, transports, technologies de l'in- 
formation et de la communication (TIC) (déterminant interne 1:4 modalités);

vi) les modèles professionnels et la sociologie agricole du MC (déterminant interne $2: 3$ modalités).

Chaque grand déterminant a fait l'objet d'une analyse systématique à des niveaux plus fins, dans laquelle les différentes variables qui le composent ont été listées et décrites selon les modalités qu'elles peuvent prendre dans le futur. La combinaison logique de ces variables détaillées, selon des hypothèses décrites dans des fiches, définit une modalité du déterminant. Par exemple, le déterminant « consommation de viande » peut prendre les modalités " écocitoyenne » ou « économe » ou « hédonique », selon que le consommateur est d'abord préoccupé par les problèmes civiques, par son budget alimentaire, ou par les problèmes de qualité du produit ou encore de goût. Les variables détaillées qui le caractérisent, «prix/budget », « fréquence de consommation », « attente et image de la viande », diffèrent dans chaque modalité unitaire, mais se combinent selon une logique d'ensemble au niveau supra.

Au final dans l'étude, toutes les modalités ont été explicitées pour faciliter la compréhension analytique d'une démarche qui repose sur une combinatoire complexe et sur l'articulation de phases complémentaires d'analyse et de synthèse (Piveteau 1995) (figure 4).

En dernier lieu, les diverses modalités de chaque déterminant externe ont été combinées pour créer des décors cohérents
Figure 4. Synthèse de la méthode employée pour élaborer les scénarios.

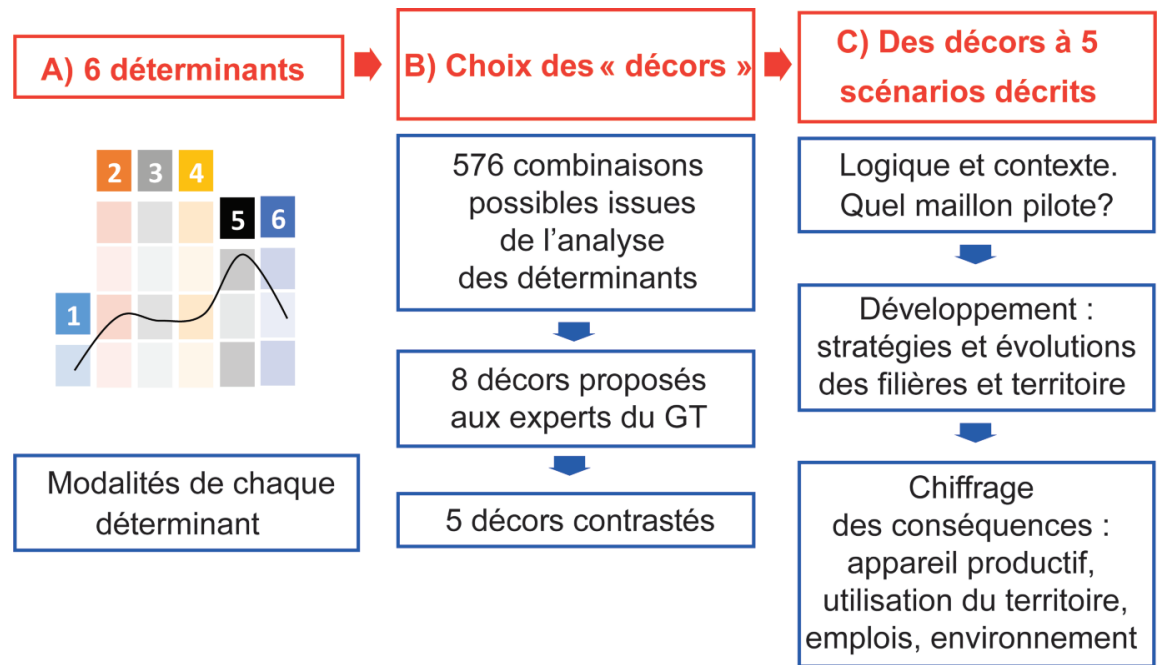

pour chaque scénario. Ces combinaisons sont récapitulées dans le tableau 2. La recherche de décors mettant en tension certains déterminants est un des objectifs de l'étude prospective car il s'agit d'envisager des situations futures plausibles qui ne tombent pas dans la caricature (dans laquelle tout irait magiquement dans le même sens, alors que l'on peut anticiper que les futurs intègreront plausiblement des dynamiques contradictoires). Huit décors cohérents, potentiellement en rupture avec le présent, ont été ainsi initialement proposés au groupe de travail. Pour ne pas multiplier inutilement les images, il s'est agi d'en choisir cinq in fine, correspondant à des hypothèses de travail intéressantes pour le groupe. Ce travail de sélection étant réalisé, le groupe de travail a été invité à anticiper les conséquences plau- sibles découlant des décors proposés, ainsi que les moyens de s'y adapter au mieux au regard des enjeux identifiés, selon une logique d'action adaptée. Ces cinq logiques, qui constituent les cinq scénarios, ont été synthétisées par un titre évocateur. Précisons que des décors « catastrophes » ont bien été identifiés, mais abandonnés du fait de leur faible intérêt pour éclairer l'action, voire de leur plausibilité (par exemple un scénario où tous les consommateurs seraient purement végétariens).

\section{e) Quantification des conséquences des scénarios}

La suite du travail a consisté à quantifier les conséquences des scénarios retenus. Un modèle spécifique décrit dans l'encadré a été développé à cette fin.

Tableau 2. Modalités des six déterminants retenues dans les cinq décors à l'origine des scénarios.

Chaque modalité (par exemple « Consommation - Économe », "Politiques agricoles - libéral vert ») est décrite en détail dans une fiche qui propose les hypothèses associées (www.ara.inra.fr/le centre/recherches).

\begin{tabular}{|c|c|c|c|c|c|}
\hline Déterminants & Décor 1 & Décor 2 & Décor 3 & Décor 4 & Décor 5 \\
\hline Consommation & $\begin{array}{c}\text { «No viande } \\
-60 \%\end{array}$ & $\begin{array}{l}\text { Econome } \\
-30 \%\end{array}$ & $\begin{array}{l}\text { Eco-citoyen } \\
-30 \%\end{array}$ & $\begin{array}{c}\text { Amateur de viande } \\
-5 \%\end{array}$ & $\begin{array}{l}\text { Econome } \\
-30 \%\end{array}$ \\
\hline $\begin{array}{l}\text { Politique Agricole et } \\
\text { Environnementale }\end{array}$ & Export sécurisé & Libéral vert & $\begin{array}{l}\text { Intervention } \\
\text { et soutiens } \\
\text { ciblés }\end{array}$ & $\begin{array}{l}\text { Réglementation } \\
\text { centralisées et régions }\end{array}$ & $\begin{array}{l}\text { Export sécurisé avec } \\
\text { assurance revenu }\end{array}$ \\
\hline $\begin{array}{l}\text { Modèles socio- } \\
\text { professionnels }\end{array}$ & « Agri-manager » & Chinois & $\begin{array}{l}\text { Producteur } \\
\text { jardinier }\end{array}$ & Familial élargi & Familial élargi \\
\hline $\begin{array}{l}\text { Aménagement du } \\
\text { territoire Massif } \\
\text { central }\end{array}$ & $\begin{array}{l}\text { Développement } \\
\text { régional efficient }\end{array}$ & $\begin{array}{l}\text { Massif inséré et } \\
\text { dualisation }\end{array}$ & $\begin{array}{l}\text { Économie } \\
\text { locale sobre }\end{array}$ & $\begin{array}{l}\text { Développement } \\
\text { régional efficient }\end{array}$ & $\begin{array}{l}\text { Massif inséré et } \\
\text { dualisation }\end{array}$ \\
\hline Filières & $\begin{array}{c}\text { Pilotage par la } \\
\text { production et qualité } \\
\text { d'origine }\end{array}$ & $\begin{array}{l}\text { Grande distribution } \\
\text { et bas coûts }\end{array}$ & $\begin{array}{l}\text { Pilotage par } \\
\text { la production } \\
\text { et qualité } \\
\text { d'origine }\end{array}$ & $\begin{array}{l}\text { Grande distribution } \\
\text { rentabilité mutualisée }\end{array}$ & $\begin{array}{l}\text { Pilotage par la } \\
\text { production }\end{array}$ \\
\hline $\begin{array}{l}\text { Changement } \\
\text { climatique }\end{array}$ & s temp & GES, $p$ & hétéroge & territoriale & \\
\hline
\end{tabular}


Encadré. Méthode d'évaluation des conséquences des 5 scénarios.

Chaque scénario a fait l'objet d'un calcul de conséquences technico-économiques des choix réalisés. Un modèle simple par étapes a été construit pour réaliser cette évaluation quantitative comparative de manière homogène sous le logiciel EXCEL. La situation 2010 est prise comme situation initiale dans tous les scénarios.

\section{Étape 1. Estimation de la surface disponible pour les ruminants}

La surface utilisée en 2010 pour les ruminants dans le MC est de 4,5 Million d'ha. Des hypothèses d'évolutions, correspondant à chaque logique interne de scénario, ont été faites sur le boisement (stabilité sauf dans le Sc1), l'artificialisation des terres (tendance actuelle ralentie dans Sc 2,4 et 5 ou très ralentie Sc 1 et 3), les cultures à visée alimentation humaine (accrues en Sc1 et 2) ou énergétiques (dans le Sc2)., La surface utilisable pour les ruminants est ainsi recalculée et adaptée à chaque scénario (de $3,0(\mathrm{Sc} 2)$ à $4,4 \mathrm{Mha}(\mathrm{Sc} 4)$.

\section{Étape 2. Calculs pour les systèmes bovins lait}

Les volumes de lait « standard » sont distingués de ceux de lait de « qualité » destinés à la production fromagère AOP. Pour les déterminer toujours selon les logiques de chaque scénario, sont définis :

- Une production laitière par vache selon le type de race (spécialisée ou mixte),

- Un taux de réforme (25 à $35 \%$ ),

- Une productivité en veaux sevrés (85 à 92\%),

- Des catégories de bovins produit et présent (âge, sexe),

- Un poids de carcasse de ces produits selon leur type,

- Un taux de croisement avec une race à viande (20 à 35\%),

- Un chargement (en UGB/ha),

- La production d'aliments (fourrages, céréales et protéagineux en pur ou mélanges).

Les effectifs devant être nourris, ainsi que les surfaces requises et leurs types (STH, Prairies Temporaires....), permettent le calcul de la surface utilisable par les autres espèces (ovins) ou productions.

Étape 3. Calculs pour les systèmes ovins laitiers puis systèmes ovins viande

Pour les ovins laitiers, nous avons estimé que la situation actuelle serait peu modifiée du fait que cette production est en place et peu évolutive dans les dernières décennies. Nous avons fixé la productivité numérique (nombre d'agneaux par brebis), le taux de réforme et les poids de carcasse, ainsi que le chargement et les aliments intra-consommés. Ce qui permet, vu la production laitière fixée, de calculer les effectifs, la quantité de viande et les surfaces utilisées par scénarios.

\section{Étape 4. Calculs pour les systèmes bovins viande}

Taille et composition du cheptel bovin allaitant sont calculées en vue d'utiliser et de valoriser la surface restante (surface pour l'élevage ruminant diminuée de celle allouée aux animaux laitiers et aux ovins).

Les paramètres fixés sont:

- Le chargement,

- Le taux de réforme (18 à $20 \%$ ),

- Le taux de sevrage des veaux par vaches (90 à 95\%),

- Les catégories d'animaux produites (âge, sexe, maigre ou engraissés, castrés ou non),

- Les poids vifs ou de carcasses.

Selon le type d'animaux produits, on estime un coefficient UGB par vache d'après les observations d'Inosys, complétées d'avis d'experts consultés. On peut en déduire les effectifs des différentes catégories animales en particulier le nombre de mères nécessaires.

En définitive, nous obtenons les états des variables suivantes:

- Les effectifs et les catégories d'animaux,

- Les volumes de viande et lait produits,

- L'utilisation des surfaces.

\section{Évaluations des emplois.}

Le nombre d'emplois directs (dans les exploitations) et indirects (amont, IAA, abattage, transformation, distribution, conseils, administration...) ont été évalués à partir des résultats du modèle, d'hypothèses faites par l'équipe projet et d'une extrapolation des travaux mené récemment par le GIS élevage demain sur ce sujet (Lang et al 2015).

\section{Évaluation du bilan environnemental :}

Les conséquences sont proposées sur la base d'une expertise de l'équipe complétée par d'autres éléments bibliographiques de façon à renseigner qualitativement les critères d'évaluation classique (cf. tableau 5). 
Les résultats quantifiés illustrent ainsi l'utilisation du sol de chaque situation de 2050, les productions dans les diverses filières concernées, mais aussi l'emploi direct et indirect associé à ces filières. Les comparaisons des évaluations chiffrées permettent de s'assurer de la plausibilité, de la cohérence des hypothèses et des contrastes importants de situations qui étaient recherchés dans la démarche de construction des scénarios.

\section{3 / Les cinq scénarios pour 2050}

Les scénarios sont présentés dans un ordre aléatoire qui ne préjuge pas de leur probabilité d'occurrence, ni d'une quelconque préférence. La logique de chacun est mise en avant tout comme les facteurs de rupture qui les ont initiés. La faisabilité du scénario repose aussi sur la mise en place d'une innovation majeure considérée comme acquise qui est précisée. Les conséquences générales sont évoquées dans cette description mais les contrastes sont analysés sur les données chiffrées.

\section{1 / Scénario « Excellence": Viande à l'herbe du Massif cen- tral, une référence d'excellence dans un contexte de forte baisse de la consommation européenne}

\section{a) Contexte}

La santé et le cadre de vie sont au cœur des attentes sociétales de 2050 qui viennent progressivement bousculer les habitudes alimentaires. En élevage de ruminants, l'abattage est une étape particulièrement sensible qui est de moins en moins acceptée par un citoyen de plus en plus déconnecté de l'agriculture. À cela s'ajoute des interrogations sur l'impact de l'élevage sur le changement climatique et la concurrence entre l'alimentation animale et humaine. Cela se traduit concrètement chez le consommateur par un rejet des viandes dites « industrielles », c'est-à-dire produites en suivant des méthodes de production intensives. Ce contexte entraine une baisse de $60 \%$ de la consommation individuelle de viande en France et en Europe. Le consommateur que l'on peut qualifier de très averti s'oriente vers d'autres sources de protéines, déjà existantes ou en développement (animales, végétales ou autres).

\section{b) Ruptures}

En réaction à la très forte baisse de la consommation les acteurs des filières du Massif central s'organisent collectivement pour ouvrir de nouveaux marchés en faisant évoluer la gamme de produits. Ils développent une marque « Massif » synonyme de naturalité. Ils valorisent les surfaces en herbe en insistant sur les différences de terroirs, base d'images facilitant le marketing des produits. Ils se dotent d'un outil commun de traçabilité, de certification et de mise en marché pour conquérir puis pérenniser des débouchés à l'international. Les produits exportés dans le monde hors du Massif sont des morceaux de viande haut de gamme de qualités garanties et adaptés à une catégorie de consommateurs mondiaux à fort pouvoir d'achat et souhaitant s'offrir une part de gastronomie française. Cet export de produits finis est rendu possible par une profonde réorganisation des moyens de production au sein des différents maillons.

\section{c) Conséquences}

À l'amont dans des élevages gérés par des " agri-managers » acteurs du marché, les cycles de production s'allongent pour engraisser les animaux localement. Le bovin allaitant producteur de viande est le grand gagnant tandis que le bovin laitier se maintient dans ses filières plus qualitatives productrices de fromages et que les ovins n'évoluent guère. Rattachés aux groupements de producteurs qui sont les moteurs de cette réorganisation, les outils de transformation deviennent extrêmement exigeants sur la qualité de leurs approvisionnements ainsi que sur les conditions d'abattage et la qualité des produits finis. Les principales solutions apportées sont un système de mesure et de contrôle de la qualité des animaux et des carcasses, une réduction des cadences d'abattage et le déploiement d'abattoirs mobiles sur de petits territoires. Ces mesures aident notamment à répondre aux inquiétudes d'une société qui même si elle ne consomme plus que peu de viande de ruminants, reste attentive aux méthodes de production employées dans les élevages qui l'entourent.

Cet effort consentis par l'ensemble de la filière Massif central pour exporter une viande de haute qualité permet de faire face au contexte intérieur négatif et de maintenir un dynamisme économique dans les territoires (emplois dans l'accompagnement, le marketing, la communication et la transformation). Pour le Massif central, ce changement de stratégie économique implique le développement de circuits de finition des animaux (essentiellement issus du troupeau allaitant), d'outils de transformation de viande de qualité supérieure et d'origine garantie, mais s'accompagne de la forte réduction des ventes en bovins maigres. Dans ce scénario, la surface agricole affectée aux ruminants se réduirait d'environ $13 \%$ avec une augmentation conséquente du volume de viande finie, notamment bovine, produite à l'aide d'un nombre de vaches allaitantes en légère réduction par rapport à la situation actuelle, et une baisse plus marquée du nombre de brebis allaitantes.

\section{2 / Scénario « Libéralisation » : Le Massif central s'adapte au sein de marchés libéralisés}

\section{a) Contexte}

Dans un monde où la libéralisation des marchés domine, la concurrence entre les bassins de production agricoles français, européens et mondiaux est forte ce qui place le Massif central en concurrence directe avec d'autres régions productrices de viande. En l'absence de contrôle des marchés ou de protection des zones défavorisées, le Massif central doit s'adapter en faisant des économies et en réduisant drastiquement ses coûts de production dans le but d'être compétitif pour approvisionner des marchés où le consommateur final mange moins de viande (baisse tendancielle de $30 \%$ de la consommation individuelle de viande de ruminants en France et en Europe) tout en étant très attentif au prix, quitte à se reporter sur d'autres viandes ou d'autres sources de protéines. Dans des filières très désorganisées, chacun des maillons et des acteurs joue de manière individuelle au gré des opportunités. En montagne, l'agriculture se recentre sur l'élevage d'animaux allaitants maigres qui reste viable économiquement grâce à l'usage extensif des surfaces en herbe. Ailleurs, la culture des céréales se développe partout où elle est envisageable, c'est à dire principalement en périphérie du Massif ou dans les plaines productives intérieures.

\section{b) Ruptures}

Peu rémunératrice, la production de bovins maigres nécessite une réorganisation du foncier, avec la constitution d'exploitations de très grande taille gérées avec très peu de main d'œuvre. Les chefs d'exploitation font appel à l'automatisation et à la robotisation pour assurer des tâches de surveillance des troupeaux, ainsi qu'à des prestataires externes. Très localement des « poches d'engraissement » persistent là où il est possible de produire des cultures destinées à l'alimentation de finition des femelles dans des ateliers spécialisés, que le marché intérieur continue à demander en priorité. Les espaces restants sont ceux où les cultures ne sont pas possibles et où les productions animales ne sont plus compétitives. La production de biomasse à partir de cultures ligneuses ou cellulosiques s'y développe ou alors les surfaces sont délaissées (friches). Dans ce contexte, la filière laitière concurrentielle (hors zones AOP) est en forte perte de vitesse et se limite aux zones intermédiaires avec cultures. 


\section{c) Conséquences}

Dans ce scénario du laisser-faire où les marchés sont libres, chaque acteur économique doit tirer son épingle du jeu. Pour le Massif central, cela implique de ne miser que sur la production très extensive de broutards accompagnée d'une compression extrême des coûts quitte à abandonner d'autres productions. L'élevage bovin allaitant s'extensifie, le nombre de vaches allaitantes pouvant diminuer de $30 \%$ d'ici 2050 . L'aval de la filière est affecté puisqu'il perd la majorité de son approvisionnement en animaux finis. Des outils de transformation disparaissent ; les grandes unités préfèrent migrer dans des régions productrices et plus facilement accessibles.

La présence du loup impacte fortement les élevages pastoraux d'ovins et le cheptel baisse fortement. En conséquence de l'abandon de certaines surfaces agricoles où le paysage se referme, des zones se retrouvent en déshérence. Le développement économique des territoires, pâtit fortement de la forte extensification et de la perte de dynamisme des pôles ruraux. Le tourisme, secteur attentif à la vitalité identitaire des différentes zones du Massif, se réduit également.

\section{3 / Scénario «AgroEco» : La viande agroécologique pour tous}

\section{a) Contexte}

Des attentes sociétales très fortes s'expriment en faveur de modes de production attentifs aux écosystèmes et respectueux des animaux et des hommes. L'agriculture dite « conventionnelle » a migré vers une agriculture « durable». Le consommateur est un " consomm'acteur écocitoyen » qui a conscience des rôles de l'élevage : à la fois responsable de pollutions mais compensées entièrement ou partiellement par une bonne gestion. Le service économique de l'élevage qui contribue à l'équilibre des territoires est reconnu et enseigné. Même si la viande est moins consommée qu'avant (baisse tendancielle de $30 \%$ de la consommation individuelle de viande de ruminant à l'horizon de 2050), elle fait toujours partie du panier d'achat du citoyen consommateur.

\section{b) Ruptures}

L'objectif est de développer des systèmes les plus efficients possibles et de répondre à des standards environnementaux exigeants. Le recours aux pratiques agroécologiques devient rapidement la norme dans le Massif central car elles minimisent les intrants. Les systèmes de production extensifs ont été faciles à convertir vers l'agriculture biologique qui se généralise. Ces systèmes sont désormais optimisés économiquement, socialement et techniquement pour jouer des diversités, des complémentarités et de la mixité entre les espèces, les produits, les labels, les territoires. Les critères recherchés d'autonomies alimentaire et énergétique poussent les éleveurs à développer sur place une part de cultures protéiques ou énergétiques tout en conservant une herbe de qualité synonyme de valeur alimentaire mais aussi de qualité environnementale. Les ressources naturelles sont ainsi utilisées plutôt intensivement afin de répondre aux objectifs d'autonomie régionale et locale qui augmentent les besoins (énergie, protéines, fourrages).

\section{c) Conséquences}

Le fait zootechnique marquant est le (re)développement de la vache mixte produisant aussi bien du lait que de la viande à partir d'une herbe jardinée à haute valeur nutritionnelle (au détriment notamment du cheptel allaitant dont le nombre de vaches pourrait chuter de $50 \%)$. Le volume de lait produit dans le Massif central augmente avec une tendance encore plus forte pour le lait destiné aux filières sous signes de qualité. Certes, ces méthodes de production ont un coût supposé supérieur mais il est en partie supporté par le consommateur et soutenu par des politiques agricoles européennes qui garantissent et protègent les marchés. La diversification du revenu des exploitations passe également par leur contribution active au mix énergétique national, notamment par la méthanisation.

Le maintien d'un tissu rural fort favorise le redéploiement des emplois dans les outils de transformation du Massif central. Les structures industrielles cohabitent avec les outils de petite taille où la production de viande à forte valeur ajoutée rentabilise les investissements consentis.

Au niveau du territoire, la surface agricole mobilisée pour les ruminants s'étend de façon conséquente $(+30 \%$ d'ici 2050$)$ et la diversité s'accentue, c'est-à-dire qu'à différentes échelles, on peut observer plusieurs types de productions et donc différents types de paysages (mosaïque) ce qui renforce l'attrait touristique.

\section{4 / Scénario «Partenariat »: La distribution alliée aux régions, partenariat pour une viande de Massif}

\section{a) Contexte}

Le Massif central étant reconnu comme une importante zone productrice de viande, ses régions (collectivités administratives et politiques) décident d'impulser une démarche de relance, et d'appuyer un partenariat constructif, équitable et reconnu entre les producteurs, les transformateurs et les distributeurs. La création et le partage de la valeur ajoutée conditionnent la réussite de ces contrats. La transparence entre les acteurs est la règle et elle s'appuie sur des outils performants pour la mesurer et l'évaluer. La consommation totale de viande rouge est stable dans le temps (très légère baisse de la consommation individuelle, compensée par la démographie). Cela favorise la réussite de cette politique régionale volontariste du Massif central qui se positionne ainsi comme un espace spécifique disposant d'une gamme diversifiée de produits de qualité issus des élevages ruminants.

\section{b) Ruptures}

Pour les acteurs territoriaux, l'objectif majeur est de promouvoir la contractualisation et de capter une partie de la valeur ajoutée sur le territoire pour maintenir un tissu socio-économique développé, garant du dynamisme rural. Pour les acteurs économiques, le but est de valoriser au mieux auprès des consommateurs, la viande du Massif, produite à l'herbe dont la qualité particulière est recherchée. La baisse de la consommation est ainsi contenue par des outils de prédiction de la qualité intégrant les attentes du consommateur, accompagnés d'une traçabilité renforcée qui le renseigne sur la totalité des étapes d'élaboration du produit (du champ à l'assiette).

\section{c) Conséquences}

Les espaces herbagers, en expansion significative, sont utilisés intensivement pour couvrir au mieux les besoins des animaux. On cherche à optimiser la ressource herbagère pour fournir une alimentation en quantité mais aussi en qualité (valeur alimentaire). Dans ces conditions, le cheptel bovin allaitant s'accroit légèrement $(+7 \%)$ et les cycles de production s'allongent (moins de mères mais plus d'animaux en finition), tandis que les autres catégories de ruminants (bovins lait, ovins) tendent à diminuer. L'amont est à l'écoute des exigences de l'aval et réactif grâce à des avancées en génétique, dans le but de produire aussi rapidement que possible une gamme d'animaux adaptés aux marchés toujours fluctuants vers lesquels ils sont fléchés (contractualisation avant même leur mise en production).

Une montée du niveau moyen de la qualité organoleptique est nécessaire pour répondre à la demande d'un consommateur toujours plus averti et connaisseur mais qui est prêt à payer un peu plus cher pour cette qualité garantie. 


\section{5 / Scénario « Géopol »: La viande géopolitique}

\section{a) Contexte}

Les tensions s'accroissent dans le pourtour méditerranéen à cause d'une forte hausse de la dépendance alimentaire de cette région $(+70 \%)$ sous l'effet conjugué de la hausse de la démographie $(+52 \%)$ et du changement climatique (Le Mouel et al 2013). L'Europe, dont l'influence est renforcée grâce à la coopération de ses membres, s'implique alors pour assurer l'équilibre géopolitique de cette région du monde. La politique européenne retrouve une place centrale dans la régulation et le développement des marchés. Avec l'ensemble des pays de l'Afrique du Nord et du Moyen-Orient, l'Europe s'accorde sur des échanges réguliers et négociés entre produits énergétiques (fossiles, puis renouvelables) et produits animaux (lait, viande), au-delà des exportations de céréales de première nécessité. Derrière les commodités laitières, les viandes de bœuf ou de mouton participent aux bouquets de produits du marché garanti trans-méditerranée.

\section{b) Ruptures}

La politique volontariste de l'Europe bénéficie directement aux régions d'élevage (principalement aux grands élevages laitiers du nord de l'Europe, y compris le grand ouest français) qui y trouvent un relai de croissance salutaire, alors qu'en interne la consommation individuelle de viande tend à baisser (-30\% d'ici 2050). Composantes de cette dynamique politique, les acteurs économiques du Massif central pèsent sur les marchés en faisant valoir leur fort degré d'organisation, notamment dans les filières de production d'animaux vivants élevés à moindre coût avec une ressource herbagère abon- dante. En renforçant sa capacité de production d'une gamme diversifiée de broutards, le Massif central s'impose comme le fournisseur majeur de ce secteur.

\section{c) Conséquences}

La force de la filière d'élevage du Massif réside dans sa capacité à s'adapter aux exigences de ce marché qui demande des formats d'animaux variables. Parallèlement les élevages ont localement besoin d'animaux rustiques et qui valorisent bien une herbe qui est l'unique source d'alimentation. Le développement d'une génétique animale maîtrisée, rapidement modulable est source de réactivité dans les changements des caractéristiques des animaux à produire. Ces animaux maigres produits dans le Massif central sont ensuite engraissés, abattus et transformés en France dans les bassins céréaliers. On observe une forte spécialisation et un fort niveau d'intégration par des structures géantes qui pilotent la production, y compris l'engraissement, et gèrent les accords avec les importateurs du bassin méditerranéen. Même si ces échanges font l'objet d'accords bilatéraux, des subventions européennes sont là pour assurer l'équilibre économique de la filière en cas de trou d'air du marché.

Les conséquences en termes d'emploi et de dynamisme économique sont plutôt négatives pour le Massif central puisque la valeur ajoutée captée par la finition et la transformation est déplacée hors de la zone. Le nombre de vaches allaitantes s'accroît fortement $(+48 \%)$ puisque les cycles de productions sont très courts (femelles allaitantes « moules à veaux ») et le nombre de brebis (allaitantes et laitières) reste stable sur une surface agricole affectée aux ruminants en augmentation de $23 \%$.

\section{4 / Les conséquences chiffrées des scénarios}

Le modèle décrit dans l'encadré a contribué à l'analyse des effets des variations des facteurs de production (par exemple le chargement animal) et des systèmes d'élevage mis en œuvre dans chaque futur possible. Cette étape grâce à ce chiffrage qui est évidemment très sensible aux choix opérés a permis de faire concrètement apparaître les contrastes. La lecture de ces chiffres doit pourtant se faire relativement, c'est à dire en comparant les scénarios entre eux et par rapport à la situation actuelle (tableau 3).

\section{1 / L'utilisation des surfaces}

Par rapport à la situation de référence (RA 2010), la place centrale des surfaces en herbe dans la surface du Massif Central est maintenue dans les cinq scénarios envisagés, et cette herbe reste la base de la couverture des besoins nutritionnels des ruminants lorsque l'on raisonne sur la base de la surface allouée aux ruminants (figure 5). En effet la production fourragère globale n'est pas réellement affectée par le changement climatique. Tous les scénarios l'intègre en effet selon les hypothèses du GIEC ${ }^{2}$ à horizon proche (2050). Les facteurs associés qui risquent d'affecter la prairie et sa production sont connus : hausse du $\mathrm{CO}_{2}$ et des températures entre 1 et $1,5{ }^{\circ} \mathrm{C}$ par rapport à la période de référence 19762005 , et augmentation de la fréquence des événements hors normes de type canicule et sécheresse (Klumpp et al communication pers.). Les deux premiers facteurs sont plutôt favorables sur la pousse de l'herbe, (Moreau et al 2008, Ruget et al 2013), mais idéalement leurs conséquences seraient à moduler localement pour affiner leurs impacts sur les

Tableau 3. Conséquences chiffrées des scénarios sur les productions du Massif central. Comparaison à la référence annuelle 2010.

\begin{tabular}{|l|r|r|r|r|r|r|}
\hline & \multirow{2}{*}{ Référence } & \multicolumn{5}{|c|}{ Scénarios } \\
\cline { 3 - 7 } & & Excellence & Libéralisation & AgroEco & Partenariat & Géopol \\
\hline Vaches laitières $(\mathrm{n})$ & 530000 & 220000 & 250000 & 720000 & 310000 & 290000 \\
\hline Lait de qualité (M I) & 440 & 440 & 440 & 570 & 520 & 440 \\
\hline Lait standard (M I) & 2470 & 1240 & 1340 & 3510 & 1680 & 1420 \\
\hline Vaches allaitantes (n) & 1800000 & 1348000 & 1030000 & 765000 & 1580000 & 2170000 \\
\hline Viande bovine (ktec) & 300 & 430 & 160 & 380 & 500 & 360 \\
\hline Brebis laitières (n) & 710000 & 710000 & 710000 & 710000 & 710000 & 710000 \\
\hline Brebis nourrices (n) & 1400000 & 845000 & 420000 & 2300000 & 840000 & 1400000 \\
\hline Viande ovine (ktec) & 50 & 30 & 20 & 70 & 20 & 50 \\
\hline Broutards du Massif (n) & 980000 & 120000 & 560000 & 130000 & 140000 & 1320000 \\
\hline
\end{tabular}


Figure 5. Utilisation des surfaces allouées aux ruminants en fonction des 5 scénarios.

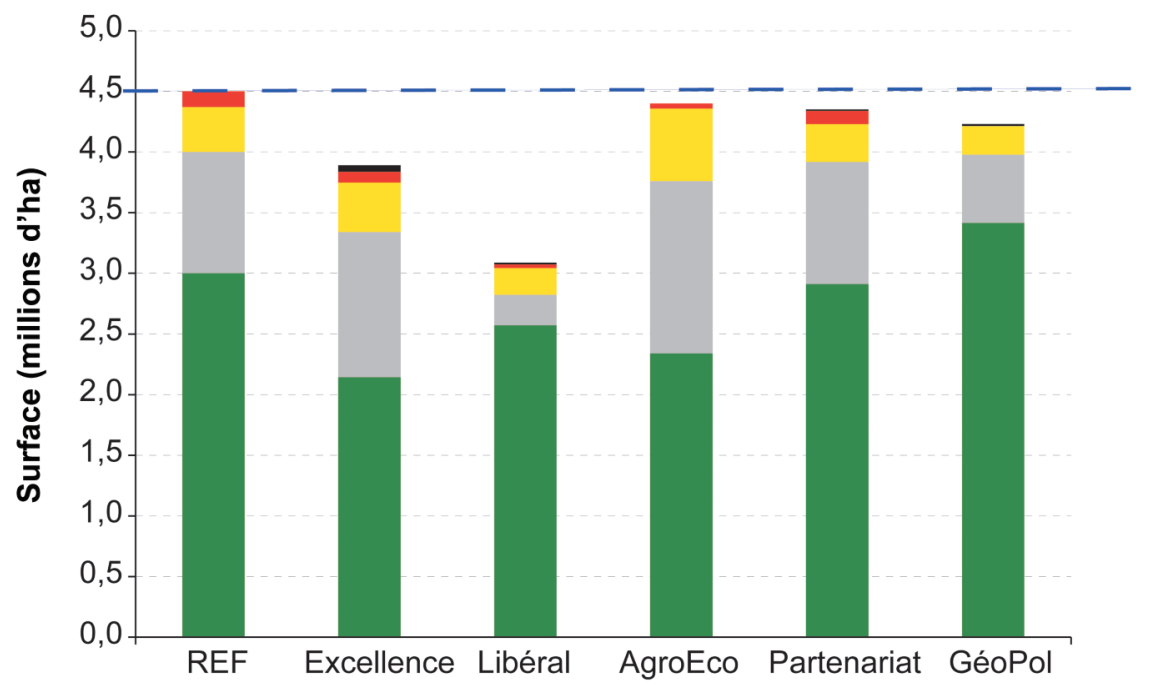

- Autres (nouvelles affectations) — Maïs fourrage-ensilage

¿ Céréales $\square$ Prairies temporaires $\square$ Surface toujours en herbe

systèmes de production. Les zones déficitaires en pluviométrie sont surtout au sud du MC (bord des Cévennes). Le reste du MC semble avoir un bilan annuel positif de précipitations par rapport à la référence, le Limousin serait la région géographique la plus positivement impactée. Pour les zones plus froides de moyennes montagnes, c'est la hausse des températures au cours du temps qui modifiera profondément les conditions de production et rendra possible des récoltes (coupe supplémentaire, maïs) parfois irréalisables aujourd'hui. L'atout " herbe », spécificité du Massif central, serait ainsi préservé à l'échéance 2050 et peut donc encore être mis en exergue : c'est un point important dans ce travail. Toutes les hypothèses considérées dans les scénarios maintiennent ensuite une majorité de STH dans la SAU ce qui n'empêche pas des contrastes importants sur la part de la SAU allouée aux ruminants, sur celle de la prairie temporaire dans la surface fourragère et sur la part des céréales. Si les scénarios 3, 4 et 5 gardent une SAU équivalente à l'actuelle, la part de celle-ci destinée aux ruminants tend à s'accroître dans les trois. À l'inverse, le scénario d'excellence (SC1) favorise les forêts et les surfaces destinées aux cultures pour l'alimentation humaine alors que, dans le scénario libéralisation (SC2), la SAU allouée aux ruminants baisse fortement en faveur de nouveaux usages agricoles des surfaces pour la biomasse énergétique ou de production de céréales (hors élevage).

\section{2 / Le cheptel et sa production dans les filières}

Les cheptels évalués en UGB présentes vont du simple au double selon les scénarios (SC2 vs SC5) (tableau 4). Ces variations résultent de la composition des troupeaux : davantage d'élèves dans le scénario excellence où la finition et l'engraissement sont adoptés et plus de vaches laitières mixtes dans le scénario AgroEco. Le scénario Géopol privilégie quant à lui des mâles maigres et les vaches allaitantes. Ces estimations ont été réalisées sans considérer finement les possibilités d'amélioration génétique des animaux.

La productivité du travail est très variable selon les types d'animaux produits et leur degré de finition. Les scénarios où les filières de production d'animaux maigres sont dominantes ont des rations UGB/UTA plus élevés.

Les filières laitières ont été prises en compte dans la modélisation des scénarios 2050 pour évaluer le nombre d'animaux d'origine laitière qui produiraient de la viande. La filière lait de brebis n'est pas impactée dans ce travail (aucune hypothèse particulière d'évolution). En lait de vache les filières «qualité, principalement destinées à la transformation fromagère sous appellation $\mathrm{AOP}$ » ont été identifiées et analysées à part. Ainsi dans les deux scénarios AgroEco (SC3) et Partenariat (SC4), les hypothèses seraient favorables au maintien voire au développement des volumes de ce lait : respectivement +30 et $+20 \%$ par rapport à la situation de référence. À l'inverse la production de lait «standard » classique baisserait dans tous les scénarios : de $32 \%$ dans le SC4 à 50\% dans le SC1. Le Massif central en concurrence avec des régions de l'ouest ou du nord de l'Europe est jugé moins compétitif sur cette filière. En revanche, dans le SC3 l'économie est avant tout locale et circulaire. À ce titre les producteurs locaux se réapproprient les consommateurs du voisinage et l'efficience des filières poussent vers des races mixtes productives en lait et en viande. La production laitière augmenterait de $42 \%$ à partir de races mixtes qui contribueraient plus fortement à la production de viande sur ces marchés essentiellement locaux.

La production de viande à partir de bovins élevés et abattus dans le Massif central, a été dissociée de celle provenant des animaux vendus « maigres » destinés à l'engraissement (en Italie, Espagne mais aussi en France hors Massif). La

Tableau 4. Utilisation du territoire et cheptel (UGB) selon les scénarios.

\begin{tabular}{|l|c|c|c|c|c|}
\hline Scénarios & $\begin{array}{c}\text { SAU Ruminant } \\
\text { (Milliers ha) }\end{array}$ & $\begin{array}{c}\text { STH } \\
\text { (Milliers ha) }\end{array}$ & $\begin{array}{c}\text { UGB } \\
\text { (milliers) }\end{array}$ & UGB/UTA & $\begin{array}{c}\text { UGB/SAU } \\
\text { Ruminant }\end{array}$ \\
\hline Référence & 4500 & 3000 & 4520 & 51 & 1,00 \\
\hline Excellence & 3900 & 2100 & 3440 & 68 & 0,88 \\
\hline Libéralisation & 3100 & 2600 & 2210 & 113 & 0,71 \\
\hline AgroEco & 4400 & 2300 & 3720 & 50 & 0,85 \\
\hline Partenariat & 4400 & 2900 & 4330 & 109 & 1,00 \\
\hline Géopol & 4200 & 3400 & 4570 & 133 & 1,08 \\
\hline
\end{tabular}

SAU = Surface Agricole Utile $; \mathrm{STH}=$ Surface Toujours en Herbe ; UGB = Unité Gros Bovin ; UTA = Unité de Travail Annuel. 
spécialisation des élevages du Massif vers la production de maigre les fragilise par rapport à l'évolution de ces marchés et est en partie à l'origine de cette prospective. Les hypothèses sont très contrastées dans les scénarios. La production d'animaux maigres resterait majoritaire dans le Massif pour le scénario Libéralisation (SC2) alors qu'elle se développerait significativement dans le SC5 Géopol. Dans les trois autres scénarios, elle serait amenée à décliner et davantage d'animaux seraient à finir d'engraisser sur le territoire du Massif, en considérant que les contraintes zootechniques et économiques liées à cette activité seront levées. Les formes d'itinéraires de finition sont cependant assez différentes et n'amènent pas les mêmes contraintes. Les taurillons sont plutôt choisis dans le SC5 Géopol alors que bœufs et génisses en cycle long sont préférés dans les SC1 et SC3. Dans le SC4 une finition sous forme de veau de lait est conservée pour des consommateurs connaisseurs.

Figure 6. L'emploi dans les filières selon les différents scénarios (emplois directs = exploitations).

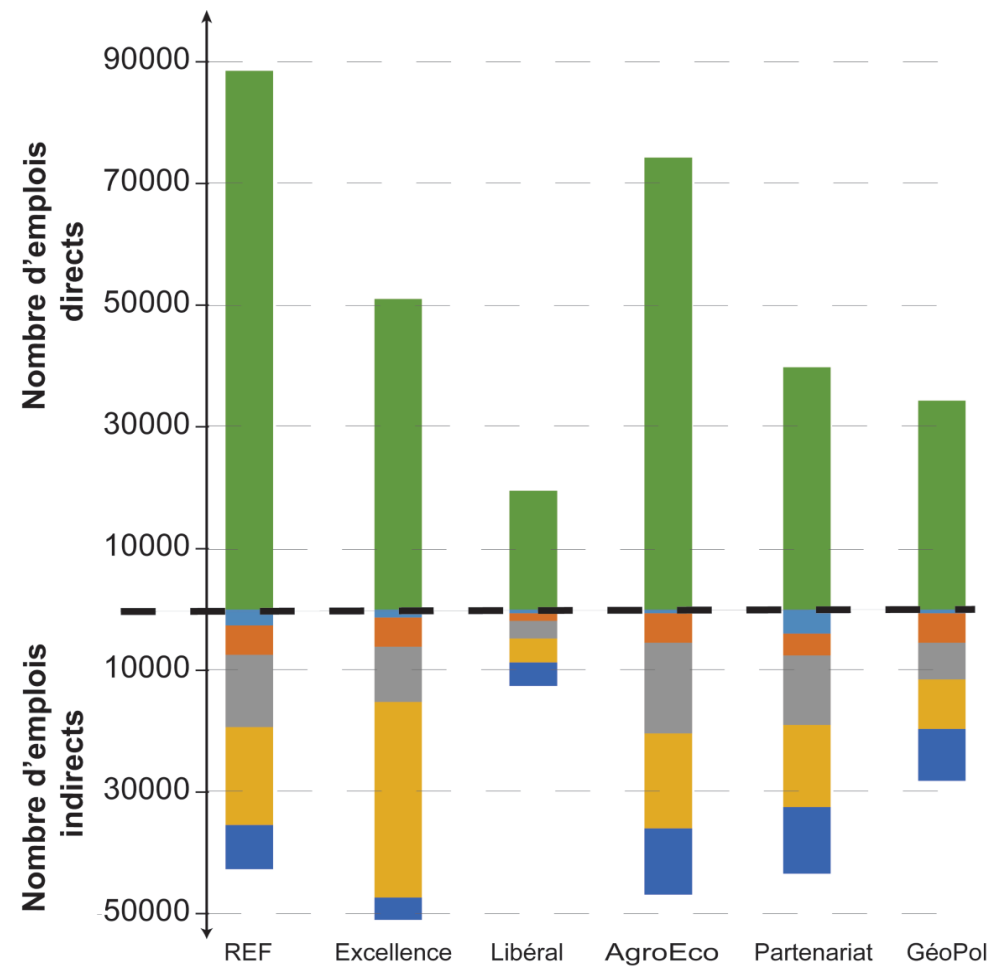

EA Ruminants Distribution $\square$ Autres biens et services

Collecte, transformation commerce, commerce de gros

Services publics et parapublics Alimentation animale

\section{3 / L'emploi agricole et dans les filières}

Toujours en baisse par rapport à la situation actuelle, l'emploi direct agricole tout comme l'emploi indirect (5 catégories dans la partie basse des histogrammes de la figure 6) pourrait fortement régresser dans certains scénarios. Dans le scénario libéralisation (SC2) par exemple où l'on recherche particulièrement les économies d'échelle, moins de 20000 emplois demeureraient dans les exploitations agricoles. À l'inverse, la baisse serait contenue dans le scénario AgroEco (SC3) qui nécessite de la main d'œuvre et conserverait 75000 emplois dans les exploitants. En dépit de la baisse très accentuée de la consommation de viande, le nombre d'emplois agricoles serait moins affecté dans le scénario de l'excellence (SC1) car la finition d'animaux demande un important savoir-faire humain. Ce même scénario enregistrerait une augmentation du nombre d'emplois indirects notamment dans la transformation et la commercialisation des produits carnés haut de gamme que le Massif central exporterait (marketing, informations internet, construction de l'image). Dans les scénarios Partenariat et Géopol, le nombre d'emplois directs serait d'environ 35 à 40000 mais ils divergent au niveau des emplois indirects. Des filières orientées uniquement vers la production d'animaux maigres (SC5 Géopol) feraient fortement chuter les emplois dans la transformation puisqu'elle ne serait alors plus nécessaire sur le territoire.

\section{4 / L'environnement}

Les impacts environnementaux des scénarios ont été analysés qualitativement via le nombre total d'animaux, le chargement moyen, les systèmes fourragers et la part des prairies dans le territoire (tableau 5).

Tableau 5. Estimation des conséquences environnementales de chacun des scénarios.

\begin{tabular}{|l|l|c|c|c|c|c|}
\hline \multirow{2}{*}{ Critères } & \multirow{2}{*}{ Indicateurs } & \multicolumn{5}{|c|}{ Scénarios } \\
\cline { 3 - 7 } & & Excellence & Libéralisation & AgroEco & Partenariat & Geopol \\
\hline GES & kg eq CO2 & ++ & +++ & ++ & + & $=$ \\
\hline $\begin{array}{l}\text { Acidification } \\
\text { Eutrophisation }\end{array}$ & $\begin{array}{l}\text { kg eq SO2 } \\
\text { kg eq PO4 }\end{array}$ & ++ & +++ & +++ & $=$ & $=$ \\
\hline Energie fossile & kJ & - & ++ & $-/+$ & - & $=$ \\
\hline Biodiversité & $\begin{array}{l}\text { STH et } \\
\text { chargement }\end{array}$ & $=$ & + & + & - & - \\
\hline $\begin{array}{l}\text { Diversité } \\
\text { paysagère }\end{array}$ & $\begin{array}{l}\text { Usage des sols }- \\
\text { haies }\end{array}$ & - & $--/++$ & +++ & - & $=$ \\
\hline $\begin{array}{l}\text { Patrimoine } \\
\text { paysages }\end{array}$ & Friche & - & -- & ++ & $=$ & $=$ \\
\hline
\end{tabular}

+/-/= : effet positif/négatif ou inchangé pour le critère par rapport à la situation de référence. 
$\mathrm{Du}$ fait de son orientation vers une agriculture agro-écologique, le scénario 3 ressort favorablement sur tous les critères analysés. À l'opposé, les scénarios 1,4 et 5 mobilisent moins les équilibres agro-écologiques et se traduisent par des impacts environnementaux soit stables, soit accentués. Dans le scénario de libéralisation (SC2), le bilan est positif pour plusieurs critères environnementaux directement liés au chargement qui dans ce scénario est très faible $(0,7 \mathrm{UGB} / \mathrm{ha}$ de SAU). Il est néanmoins très défavorable si l'on regarde la dégradation des paysages résultat d'enfrichement dans de nombreuses zones intermédiaires.

\section{5 / Discussion sur les choix méthodologiques et leurs effets}

\section{1 / Élaboration et choix des décors}

Lorsque l'équipe projet a proposé des combinaisons de modalités des déterminants externes pour construire des décors de scénarios, chaque décor a été pensé en partant du déterminant « consommation de viande à l'échelle européenne " . Ce déterminant comporte 4 modalités qui ont été réparties dans 4 décors. $\mathrm{Ce}$ premier choix s'est imposé naturellement à l'équipe projet car il semblait important de partir de cette extrémité de la filière (la demande) pour mieux tenir compte du contexte et des attentes du consommateur. C'est la demande qui au final structurerait l'offre. C'est le consommateur qui oriente la filière. La place prépondérante prise récemment par la consommation « commode » et l'augmentation récente de la consommation de viande hachée viennent à l'appui de ce choix. En outre, c'est bien la consommation qui crée de grosses inquiétudes dans la filière.

Parallèlement au déterminant « consommation ", on peut relever le choix de ne proposer qu'une modalité pour le déterminant externe " changement climatique ». Les experts et les études mobilisées indiquent qu'à l'horizon 2050 le changement climatique est quasiment certain, les scénarios climatiques ne commençant à diverger qu'à partir de 20402050. Les conséquences de ce phénomène en termes d'augmentation de la fréquence des aléas (sécheresses notamment), de changements dans la nature des cultures à lieux et altitudes données (céréales dans la planèze de St Flour par exemple), sont quant à elles entièrement intégrées aux réflexions.

Si parmi les combinaisons possibles certaines semblaient très peu plausibles (modalités incompatibles entre elles), il en restait un trop grand nombre pour qu'elles soient toutes étudiées et surtout contrastées. Avec pour objectif de produire des scénarios en nombre limité, l'équipe projet a sélectionné des décors qu'elle a jugés « satisfaisants intellectuellement 》 mais aussi « possibles et attendus » par une majorité d'acteurs. D'autres décors étaient donc possibles et cette étape de sélection est bien évidemment très sensible sur le résultat final. La validation par le comité de pilotage et la traçabilité du raisonnement sont les seuls justificatifs possibles. Toutefois, ces décors n'ont eu que le statut de propositions puisque le groupe de travail a eu ensuite l'opportunité de les amender pour les transformer en scénarios.

Il faut également noter que le champ des possibles qui ont été conservés dans chaque cas analysé, reste assez stable sur des hypothèses macro-politiques. L'UE ou son équivalent demeure quel que soit le scénario. L'hypothèse de crise majeure qui conduit à l'effondrement de l'économie conventionnelle n'a pas été conservée même dans le scénario 3 qui repose largement sur une nouvelle économie avec relocalisation de la production et des échanges. Il n'a pas non plus été envisagé de crise sociétale forte. Ainsi, le comité de pilotage a souhaité éviter les scénarios dits « catastrophes », c'est à dire ceux où aucune marge de manœuvre n'était laissée aux acteurs du Massif central et même dans le scénario 1, il y a encore de la viande consommée (environ $20 \mathrm{~g}$ de viande /habitant/jour - un steack par semaine - ou $10 \mathrm{~kg}$ équivalent carcasse /habitant/an). Tous les groupes qui ont donné leur avis sur ce point ont convergé vers l'idée qu'il n'y avait pas de raison évidente d'aller plus loin que les ruptures envisagées qui sont déjà bien réelles.

\section{2 / Analyse des impacts}

L'étude prospective a été réalisée sur le Massif central, entité administrative définie par la loi Montagne de 1985. Ce périmètre comporte plusieurs grands bassins d'activité homogènes dans leur fonctionnement. La manière de traiter ces territoires (découpage géographique ou économique) et d'analyser leurs interrelations peut modifier la perception des équilibres d'avenir. Nous avons considéré les quatre bassins distingués dans l'étude SIDAM-Idele (Sanne et al 2013) (figure 1), en retenant leurs invariances géographiques et fonctionnelles à l'échelle de 35 ans. Il n'a pas été fait d'hypothèses d'avenirs différenciés selon les gouvernances des nouvelles régions. Il est pourtant réaliste de penser que les développements territoriaux sont interdépendants voire en compétition. Une analyse plus fine par micro-scénario territorialisé aurait peut-être mieux apprécié cet aspect compétitif et décrit des évolutions contrastées des secteurs marchands, et non marchands (malgré la difficulté de les appréhender) intra Massif central. Toutefois nous retenons les convergences de scénarios avec la prospective " élevage allaitant de Saône et Loire » réalisée en 2009. Un décor axé sur la qualité maîtrisée des produits et un autre sur le fort agrandissement des structures dans un marché libéral s'y retrouvent. Chacun a des conséquences particulières sur cette zone centrale du bassin Charolais.

\section{3 / Sensibilité des scénarios à certaines hypothèses}

Les résultats chiffrés reposent sur des hypothèses de SAU disponible en 2050 calculées tendanciellement. Les besoins fourragers par tête sont issus des références actuelles observées à partir du RA 2010 (typologie Inosys réseaux d'élevage). L'intégration de l'amélioration génétique sur cet indicateur de besoins n'a pas été envisagée. Les évolutions des animaux et de l'efficience d'utilisation des aliments auraient aussi pu faire l'objet de prévisions tendancielles, mais c'est un niveau de productivité global par hectare choisi en cohérence avec la logique de chaque scénario qui a été préféré. Aussi le modèle de calcul utilisé chiffre et illustre les principaux indicateurs (de production, d'emplois et d'utilisation du territoire) qu'il convient d'interpréter dans le but de révéler les contrastes issus des réflexions propres à chaque scénario. Ce modèle créé pour chiffrer les scénarios est sensible aux hypothèses émises.

\section{a) Hypothèses d'évolution de la consom- mation}

L'analyse du passé a mis en avant des tendances récentes, mais que l'on peut déjà qualifier de lourdes. La baisse de la consommation individuelle de viande de ruminants est continue depuis plus de 30 ans. Les évènements économiques et les attentes sociétales laissent penser que cette tendance va se poursuivre. Dans les groupes de travail, la question a essentiellement été d'évaluer le niveau de baisse de la consommation individuelle et les modes de consommation qui y seront associés. Les scénarios $(2$, 3 et 5 ) reposent sur une baisse équivalente à la tendance moyenne depuis 20 ans (Combris 2003) soit - 0,8\% par an arrondie à $-30 \%$ en cumulé sur les années qui nous séparent de 2050. Récemment le "World Cancer Research Fund 》 a indiqué qu'un apport de plus $70 \mathrm{~g} / \mathrm{j}$ de viande " rouge » n'est pas souhaitable (Lecerf 2014). Bien que la consommation moyenne française soit déjà inférieure (50 g/habitant/j de viande « vraie » en 2010 et non en équivalent carcasse 
comme c'est souvent indiqué, Crédoc 2012) ce message a été fortement relayé dans les médias ce qui risque d'affecter durablement les habitudes alimentaires du consommateur qui se situent dans la moyenne de consommation nationale. Les baisses les plus fortes (- 60\% SC1) ne seraient donc pas le fruit de recommandations nutritionnelles, mais bien celui d'une forte évolution de la perception de la viande et de l'image de l'élevage par les consommateurs. À l'opposé le scénario 4 stoppe cette tendance par une forte volonté des acteurs.

\section{b) Emplois}

L'évaluation du nombre d'emploi est entièrement dépendante des résultats nationaux produits par le « GIS Élevage Demain » dans son travail sur les impacts de l'élevage (Lang et al 2015). Ces travaux accompagnés d'une série d'hypothèses localisées (par exemple le nombre d'emplois dans les abattoirs en lien avec le tonnage traité) ont permis d'estimer par calculs simples un nombre d'emplois directs dans les exploitations d'élevages et d'emplois indirects dans le reste des divers maillons des filières. La quantification précise de l'étude nationale au périmètre « Massif central » en repartant des bases de données départementales aurait nécessité un travail hors de portée dans le cadre de l'étude, l'ordre de grandeur des contrastes étant suffisant pour les besoins de la démonstration.

\section{c) Utilisation du territoire}

L'utilisation des surfaces est faite par chaque système de production (bovin laitier, allaitant, ovin laitier, allaitant) à partir d'une ventilation du nombre d'UGB sur la base de cas observés dans les données statistiques du réseau Inosys. Cette étape a permis de mieux qualifier la composition des surfaces agricoles allouées aux ruminants d'une manière très globale à l'échelle du Massif central.

\section{6 / Quels enseignements tirer de la démarche?}

Cet exercice de prospective apporte de nouvelles visions du futur partagées avec les acteurs souhaitant mettre en place une démarche stratégique volontairement anticipatrice. Mais il faut en parallèle évaluer la " portée » des scénarios. En positif, il faut considérer le fait d'avoir posé des repères inédits pour un débat sur l'avenir qui malheureusement se nourrit souvent de projections trop partielles, d'études à court terme et d'intuitions rarement creusées. Dans les limites de l'exercice il est clair que la recherche d'une approche globale qui mobilise des analyses économiques, politiques, environnementales, techniques et sociologiques, ne peut être qu'imparfaite. Pour être appréhendables et lisibles, les éléments produits simplifient au-delà de ce que l'on voudrait, et les hypothèses retenues sont toujours discutables et pourraient toujours être mieux étayées. La complexité de la démarche invite à une réelle modestie quant à ce qu'on a pu réellement embrasser au regard de tout ce qui serait nécessaire. Malgré ces réserves quelques enseignements nous semblent pouvoir être mis en avant, face à des questions souvent posées :

- Bien que la croissance de la population mondiale (et notoirement non européenne) soit souvent annoncée comme un gage de la pérennité des filières de ruminants, rien ne garantit qu'elle n'assure des débouchés à long terme à la production de viande du Massif central.

\section{L'équation :}

croissance de la population mondiale à $2050 \times$ hausse de l'alimentation carnée (hors Europe) $=$ augmentation de l'offre européenne

apparaît trop simpliste sur la base de l'analyse des échanges internationaux et des études de coûts. C'est pourtant à l'échelle européenne qu'il faut raisonner le devenir du Massif central, autant pour les gros volumes en vif destinés à l'engraissement que pour la viande commercialisable car les concurrences seront fortes. C'est aussi à l'échelle mondiale que peuvent se trouver de nouvelles opportunités de valorisation des produits du territoire.

- À l'horizon 2050, le changement climatique ne semble pas de nature à bouleverser la production primaire à l'échelle du Massif, en tout cas pas au-delà de ses manifestations actuelles que l'on peut commencer à pressentir (augmentation de l'occurrence des événements extrêmes). Dans nos filières, le changement climatique n'écrase pas encore, à cet horizon de temps, les autres variables motrices d'évolution sociétales, politiques et économiques. Cependant, l'importance de la ressource herbagère pour les productions, leur autonomie alimentaire, leurs coûts de production, impose une vigilance forte sur ce point climatique et la maîtrise raisonnée des risques.

- Des pistes d'évolution et des marges de manœuvre existent pour les filières du Massif central, même dans des contextes défavorables à l'horizon 2050. En jouant sur les projets d'exploitation, sur leur équilibre technico-économique, sur l'organisation des filières, sur les partenariats internes ou externes, il est possible de proposer des projets alternatifs dans lesquels la valorisation de l'herbe reste centrale. Mais, elle s'appuie sur des modalités très différentes des autres zones herbagères plus intensives d'Europe et repose ainsi sur les caractéristiques spécifiques de la prairie de moyenne montagne, sa diversité floristique et son utilisation souvent extensive, qu'il faudra valoriser par des animaux bien adaptés. L'appréciation fine des services rendus par l'élevage du Massif rentre bien dans cette valorisation potentielle.

- La construction de l'offre en viande (et en lait) doit rejoindre la demande d'un consommateur qui aura toujours le dernier mot. L'enjeu central est donc d'inscrire la production de viande du Massif central dans un équilibre complexe et construit, à la fois quantitatif et qualitatif, et de la positionner par rapport à d'autres zones concurrentes, en Europe et dans le monde. Il faut produire ce que demandent les marchés, mais on peut aussi tenter d'orienter cette demande. Ce positionnement de l'offre considère que modes de production et provenances importent, et qu'il y a lieu de jouer sur les divers registres de la qualité (organoleptique, nutritionnelle, environnementale). La régularité de l'offre est souvent soulignée comme une entrave au développement d'innovations : ce point doit être raisonné au démarrage de la construction collective.

- La qualité globale des produits et de l'offre en général, demeure un objectif majeur. Elle s'évalue sur des critères précis et explicites, se communique sur ces mêmes critères : elle ne se décrète pas! Les stratégies à long terme impliquent ainsi une organisation d'acteurs renforcée, sans pour autant se référer à l'image d'un unique projet fédérateur. $\mathrm{Ce}$ positionnement qualitatif implique d'envisager différents niveaux de fédérations des collectifs pour jouer des complémentarités territoriales et des différents acteurs des filières. L'analyse territoriale par bassin de ces stratégies est à faire pour les accompagner. L'engagement d'acteurs internes au Massif, mais aussi externes, pour construire ces partenariats définira l'image du futur.

- Pour fédérer l'ensemble de la filière, les acteurs qui interviennent en son sein doivent être attentifs, au-delà des aspects économiques, à l'évolution des attentes des producteurs sur les registres socioculturels : les exploitants qui s'installent aujourd'hui et s'installeront demain ont et auront des attentes plus proches de l'ensemble de la société. À cet égard, les évolutions sociétales et les changements culturels repérés dans les différents scénarios doivent être appréhendés de manière ouverte.

- La formation des futurs acteurs doit considérer les capacités d'évolution des filières. On peut et on devra encore plus " faire différent » pour identifier et 
valoriser les produits qui véhiculent l'image partagée du Massif. La capacité d'analyse pour innover et s'adapter aux contraintes évolutives diverses doit faire partie des aptitudes à acquérir dans les formations à l'élevage.

\section{1 / Les innovations à mobiliser}

Pour accompagner au mieux les futurs souhaités par les acteurs du Massif, les connaissances scientifiques et leurs applications sous forme d'innovations doivent être mises en œuvre. Ainsi les principales innovations mises en avant et considérées comme acquises pour rendre crédibles chaque scénario portent :

i) sur les animaux et leurs aptitudes pour inviter à disposer d'une génétique animale maîtrisée dans ses différentes fonctions et surtout qu'elle soit le plus réactive possible et plus rapidement mobilisable. Il faut accroître les connaissances (phénotypage) vis-à-vis de fonctions jusque-là considérées comme secondaires ;

ii) sur la connaissance de la ressource herbagère pour mieux raisonner la gestion et la résilience du système fourrager, au travers des diverses utilisations de l'herbe, ressource pâturée ou stockée. La prairie permanente restera majoritaire, et doit se raisonner en fonction des avancées sur la prairie cultivée. De nouveaux mélanges de variétés et d'espèces fourragères adaptées aux conditions locales (par exemple la chicorée) complèteront en effet la palette des solutions possibles dont le maïs qui pourra être cultivé en altitude et répondre à des conditions de printemps sec et été humide comme lors de l'année 2011;

iii) sur la gestion des grands effectifs animaux en réduisant les coûts de production et en disposant de moyens adéquats pour le faire tout en maîtrisant le bien-être des animaux et leur docilité. À ce titre deux pistes sont clairement dégagées qui ne sont pas incompatibles, celle de l'automatisation robotisation des tâches d'élevage de base, et celle d'une information claire simple et efficace, disponible en temps réel sur l'état du troupeau des animaux et des ressources au sens large. Cette information mise en forme doit aider à la prise de décision et non inonder d'indices variés incohérents entre eux les divers participants des filières ;

iv) sur la maîtrise de la gestion de la qualité globale des différents produits proposés au consommateur. Les niveaux d'exigence ne seront pas les mêmes s'il s'agit d'une viande destinée à un marché de luxe (SC1) ou à un marché plus classique (SC4), mais le schéma doit être raisonné de la même manière pour s'adap- ter. Il doit inclure les différentes dimensions de la qualité (organoleptique, nutritionnelle, sanitaire, image...) et les différents maillons de la chaîne de chaque filière (production, abattage, transformation, distribution, consommation). Il devrait aussi être à même de proposer un raisonnement transparent, support de la construction de la valeur ajoutée des produits vendus ;

v) sur l'augmentation de l'efficience des systèmes que peuvent amener des pratiques reposant sur les principes de l'agroécologie. Cela suppose d'identifier les gains potentiels et comment peut-on les développer et donc les mesurer, et à quelle échelle de temps. Il convient donc de mieux comprendre les processus agroécologiques à mettre en œuvre et savoir les piloter. À cette fin, la mobilisation des techniques fines de mesures, d'analyses d'images et de robotiques est très certainement à mettre en œuvre ;

vi) sur le cracking de la biomasse végétale et de l'herbe. Il faut en connaitre les potentialités, les limites et surtout les impacts notamment environnementaux car si des surfaces se libèrent et si la transmission générationnelle se complique c'est vers cette possibilité que les territoires se tourneront.

\section{2 / De voies de recherches à conforter}

L'exercice de prospective a ainsi montré que, dans plusieurs domaines, un approfondissement des connaissances était nécessaire pour répondre à une large gamme de conditions externes ou internes aux exploitations et aux filières d'élevage. Les domaines principaux adressés par les scénarios de l'étude portent sur les ressources, les conditions d'élevage et leurs conséquences agro-environnementales, les produits, leurs transformations, la consommation et, plus généralement, les conditions économiques et sociales de l'activité d'élevage. Ces questions, qui sont souvent déjà au cœur des recherches actuelles, peuvent être à la base de nouveaux projets ou dispositifs de recherche :

\section{a) Ressources alimentaires des animaux} et systèmes fourragers

L'herbe et sa bonne utilisation sont requises dans tous les scénarios. Tous ne nécessitent pas les mêmes pratiques de gestion des surfaces fourragères, ni la même maîtrise des itinéraires techniques. Chaque petite région demande des pratiques adaptées et c'est d'autant plus vrai que la pousse de l'herbe dépend entièrement des conditions climatiques (forcément aléatoires) en interaction avec les conditions de sol. L'étude met en avant l'importance de la connaissance fine des ressources par l'utilisation croisée des données disponibles, associée à leurs bonnes gestions par le développement de nouveaux outils qui aident à en tirer le meilleur parti.

\section{b) Animaux}

Dans les milieux du futur, résilience aux aléas, rusticité des femelles, docilité et défense vis-à-vis des prédateurs tout comme les aptitudes variées à tirer parti du pâturage seront des qualités animales requises en liaison avec la productivité et la qualité des produits. Les compromis entre efficience, robustesse et qualité sont encore à construire, mais, pour ces questions, la sélection des animaux grâce à des outils de la génomique va apporter des moyens nouveaux. Le partage des objectifs de sélection par des collectifs élargis ressort comme un défi nécessaire, en adaptant bien évidemment cette démarche aux spécificités des races et aux pratiques émergentes.

\section{c) Environnement}

La prairie, milieu d'élevage des ruminants, restera le garant d'un impact environnemental favorable. Dans les scénarios orientés vers la qualité ( $\mathrm{SC} 1, \mathrm{SC} 3$ et SC4), on insiste sur le lien entre image des filières, produits et attentes du consommateur. Des évaluations devront être conduites sur l'impact environnemental des productions, en considérant notamment les évolutions de la biodiversité, face au changement climatique. Cette étude rappelle que des indicateurs fiables et simples sont à mobiliser pour construire une évaluation environnementale multicritère.

\section{d) Qualité des viandes (types)}

La différenciation souhaitée des produits du Massif imposera une meilleure connaissance et maîtrise de la qualité gustative et nutritionnelle des viandes mises en marché. Au niveau de l'offre, ce défi devra se gagner par la concertation active de toutes les filières en recherchant de nouveaux indicateurs qui enrichissent les modèles de prédiction et en mobilisant de nouveaux outils de suivi individuel de la qualité. Au-delà, l'étude propose une construction partenariale de la qualité qui mobilise des jeux d'acteurs différents. Elle met en avant la bonne connaissance des déterminants de la demande et de leurs hiérarchies en France et en Europe, mais aussi vis-àvis de consommateurs potentiellement plus éloignés.

\section{e) Efficience organisationnelle des filières}

Les filières reposent actuellement sur les aides publiques à la production. Peuvent-elles s'en passer et quelles seraient 
leurs nouvelles organisations ? Quels gisements de réduction des charges permettraient, en concertation, de rendre la situation des producteurs moins périlleuse sans grever pour autant les autres maillons des filières? Les innovations matérielles et technologiques sont à évaluer, tout comme doivent l'être les innovations organisationnelles. L'analyse de faisabilité d'un abattage à la ferme dans les meilleures conditions sanitaires et de stress des animaux en est un exemple cité dans cette étude. Le ciblage en amont des techniques majeures à faire évoluer peut se construire et se partager au sein de « laboratoires vivants ».

\section{f) Économie et sociologie des productions et de la consommation}

Au niveau de l'économie de l'exploitation, la simulation de l'évolution des facteurs de production, au sein de différents types d'organisation (exploitations individuelles ou collectives) et de projets d'exploitation permettra de précéder les questions qu'en particulier le changement générationnel et la structure toujours complexe des marchés fonciers vont induire. Ces projets devront s'enrichir d'une meilleure connaissance des ressorts socio-économiques de la consommation de viande dans les différentes composantes de la société.

\section{g) Vers de nouveaux dispositifs de concep- tion et d'évaluation des innovations}

Au-delà des travaux de recherche mentionnés précédemment, et de manière à les intégrer à l'échelle de l'exploitation et des filières, il serait souhaitable de réfléchir au développement d'un outil collectif associant recherche, développement et formation basé sur des démarches participatives et favorisant la conception et la diffusion d'innovations à l'échelle du territoire. Le modèle des « laboratoires vivants » permet de répondre à cette attente. Il aurait pour finalité l'efficience et la résilience de nos filières viandes du Massif.

\section{Conclusion}

Ce travail prospectif original montre que dans les diverses versions des futurs plausibles, des stratégies d'adaptation des filières viandes sont toujours possibles, même lorsque les décors sont contraignants. Dans les scénarios, les collectifs d'acteurs « moteurs » des filières diffè- rent et leurs modes d'organisation affectent les bassins de production. Mais la gestion adaptée et raisonnée des prairies et la garantie de la qualité des produits viande sont des points sensibles incontournables dans tous les cas abordés. L'étude ne propose pas de " probabilisation » des cinq scénarios qu'il faut percevoir comme des futurs possibles décrits pour pousser à la réflexion. Mais en proposant cinq scénarios contrastés qui chacun posent des questions et peuvent interpeller les acteurs économiques et territoriaux du Massif central, cette étude peut aider à prendre des décisions stratégiques idéalement concertées et positives pour l'avenir.

\section{Remerciements}

Les auteurs remercient particulièrement J.Y. Bechler et J.B. Coulon pour leur forte implication dans le démarrage et la réussite de l'étude, ainsi que les 21 membres du groupe de travail, les 7 porteurs d'enjeux du comité de suivi, et tous les responsables qui ont accepté interview et visites. Au total plus de 50 personnes qu'on ne peut toutes nommer ici individuellement.

\section{Références}

Agreste, 2010. Service de la statistique et de la prospective du ministère de l'agriculture. L'utilisation du territoire entre 2006 et 2009 , Agreste Primeur, 246, 4p.

Agreste, 2015. Statistique Agricole Annuelle. https://stats.agriculture.gouv.fr/disar/faces/rep ort/tabDocBySource.jsp

Agreste RICA, 2014. https://stats.agriculture. gouv.fr/disar/faces/report/tabDocBySource.jsp

Auvergne Limousin, 2010. Étude des filières bovins lait à l'échelle du Massif Central http://www/agriculture.gouv.fr/ministere/situation-de-la-filiere-laitiere-du-massif-centralperspectives-davenir/

Combris P., 2003. Cinquante ans de consommation de viande et de produits laitiers en France. Renc. Rech. Rum., 10, 327-330.

Credoc, 2012. Évolution de la consommation de viande en France Les nouvelles données de l'enquête « Comportements et Consommations Alimentaires en France » (CCAF) 2010 http://www.credoc.fr/pdf/Sou/Consommation viande CCAF2010.pdf

Dodane C., 2009. Les nouvelles forêts du Massif central : enjeux sociétaux et territoriaux. Ces hommes qui plantaient des résineux pour éviter la friche. École normale supérieure Lettres et Sciences Humaines - ENS-LSH Lyon, thèse de doctorat en géographie.

Dumont B., Dupraz P., Aubin J., Benoit M., Bouamra Z., Chatellier V., Delaby L., Delfosse C. Dourmad J.Y., Duru M., Frappier L., Friant-
Perrot M., Gaigné C., Girard A., Guichet J.L., Havlik P., Hostiou N., Huguenin O., Klumpp K., Langlais A., Lemauviel-Lavenant S., Le Perchec S., Lepiller O., Meda B., Ryschawy J., Sabatier R., Veissier I., Verrier E., Vollet D., Savini I., Hercule J., Donnars C., 2016. Rôles, impacts et services issus des élevages en Europe, INRA, France. Expertise disponible sur http://institut.inra.fr/Missions/Eclairer-lesdecisions/Expertises/Toutes-1esactualites/Roles-impacts-et-services-issus-deselevages-europeens

IPAMAC, 2011. Trame écologique du Massif central, Rapport de synthèse.

Lang A., Dupraz P., Tregaro Y., Rosner P.M., Perrot C., 2015. Les emplois liés à l'élevage français. Etude GIS Elevage demain, Actes des $9^{\text {emes }}$ Journées de Recherches en Sciences Sociales Nancy, 10-11décembre 2015. https://www.giselevages-demain.org/Actions-thematiques/ Emplois-lies-a-1-elevage/Synthese-Lesemplois-lies-a-l-elevage-francais

Le Mouël C., Forslund A., Marty P., Manceron S. Marajo-Petitzon E., Caillaud M.-A., Schmitt B., 2015. Le système agricole et alimentaire de la région Afrique du Nord - Moyen-Orient à l'horizon 2050 : projections de tendance et analyse de sensibilité. Rapport final d'étude pour Pluriagri, Paris et Rennes : INRA (DEPE \& SAE2), 134p. Disponible sur http://institut.inra.fr/Missions/ Eclairer-les-decisions/Etudes/Toutes-les-actualites/Pluriagri-Afrique-du-Nord-MoyenOrient-2050-Dependance-alimentaire

Lherm M., Agabriel J., Devun J., 2017. État des lieux et évolutions de la production bovine allaitante en France et dans trois pays européens. In : Elevage bovin allaitant. Agabriel J., Renand G., Baumont R. (Eds). Dossier, INRA Prod. Anim., 30, 93-106.

Moreau J.C., Ruget F., Ferrand M., Souverain F., Poisson S., Lannuzel F., Lacroix B., 2008. Prospective autour du changement climatique : adaptation des systèmes fourragers. Renc. Rech. Rum., 15, 193-200.

Piveteau V., 1995. Prospective et territoire : apports d'une réflexion sur le jeu. Cemagref éditions, collection Gestion des Territoires, $\mathrm{n}^{\circ} 15$. Rieutort L., Ryschawy J., Doreau A., Guinot C., 2014. Atlas de l'élevage herbivore en France. Éd. Autrement Paris, France, 96p.

Ruget F., Durand J.L., Ripoche D., Graux A.I., Bernard F., Lacroix B., Moreau J.C., 2013. Impacts des changements climatiques sur les productions de fourrages (prairies, luzerne, maïs) : variabilité selon les régions et les saisons. Fourrages, 214, 99-110.

Ryschawy J., Tichit M., Bertrand S., Allaire G., Plantureux S., Aznar O., Perrot C., Guinot C., Josien E., Lasseur J., Aubert C., Tchakerian E., Disenhaus C., 2015. Comment évaluer les services rendus par l'élevage? Une approche méthodologique sur le cas de la France. INRA Prod. Anim., 28, 23-37.

Sanne E., Brouard S., Boulleau T., 2013. Définition des stratégies et des actions à mettre en œuvre pour conforter les filières viande bovine du Massif central. Rapport d'étude SIDAM Massif central, Institut de l'élevage. 
Service de la statistique et de la prospective du ministère de l'agriculture, 2010. L'utilisation du territoire entre 2006 et 2009. Agreste Primeur, $246,4 p$.
Typologie INOSYS, 2015. Fiches Auvergne "Inosys-réseaux d'élevage » : Une nouvelle lecture de la diversité des systèmes d'exploitation. http://www.myinosys.fr/index.php?id=1187
Veysset P., Benoit M., Laignel G., Bébin D., Roulenc M., Lherm M., 2014. Analyse et déterminants de l'évolution des performances d'élevages bovins et ovins allaitants en zones défavorisés de 1990 à 2012. INRA Prod. Anim., 27, 49-64.

\title{
Résumé
}

La baisse de la consommation individuelle de viande, le réchauffement climatique, le vieillissement de la population d'éleveurs, et la forte dépendance des élevages aux aides publiques, sont autant de facteurs de risques pour l'avenir des filières viandes de ruminants du Massif central. Cette étude prospective décrit cinq scénarios plus ou moins favorables de leurs futurs possibles à l'horizon 2050. Elle a été réalisée pour que les acteurs les anticipent et mettent en œuvre des stratégies d'évolution adaptées. Les cinq scénarios résultent de logiques d'évolutions très contrastées. Ils ont été construits par un collectif impliquant vingt experts dont les compétences couvraient les enjeux identifiés par les acteurs économiques et territoriaux du Massif (les porteurs d'enjeux). Les variables qui modifient l'évolution de ces enjeux ont été organisées en déterminants, dont chaque modalité a été décrite précisément puis combinée pour étayer les cinq futurs. Chacun illustre ainsi des logiques cohérentes de fonctionnement et des utilisations du territoire bien contrastées. Leurs conséquences ont été quantifiées par une démarche originale alliant expertise, analyse bibliographique et modélisation en s'appuyant sur des indicateurs de production, d'emploi et de bilan environnemental. La gestion adaptée et raisonnée des prairies et la garantie de la qualité des produits viande sont des points sensibles et incontournables dans tous les scénarios. L'analyse transversale des résultats contrastés montre également que des opportunités peuvent se révéler intéressantes pour l'avenir. Elles seront à saisir par les partenaires des filières et à faire émerger avec le concours des différents collectifs d'acteurs du territoire « Massif central ».

\begin{abstract}
Foresight study of the ruminant meat sector in the Massif Central area of France in 2050

This foresight study describes five 2050 contrasting scenarios of possible futures for ruminant meat sectors of the Massif central area in order to help its economic players to adapt and to anticipate. The study deals with the present recurring questions of meat production systems sustainability, according to the risks of a drop in individual meat consumption, global warming, age structure of farmers, and the high level in subsidies. The five scenarios were constructed thanks to a collective expert research approach, with regards to the issues identified by the Massif's stakeholders. With its own coherence and rationale, each scenario highlights possible consequences on production, jobs and labor, environmental assessment and land uses. These were estimated by an original approach combining expertise, literature review and modeling. An adapted grassland management and the establishment of a meat quality guaranty are the key sensitive issues in all five scenarios. The cross-analysis of these contrasting results also shows what stake-holders could do in order to seize upcoming opportunities.
\end{abstract}

CERLES A., LHERM M., POUX X., AGABRIEL J., 2017. Étude prospective des filières viande de ruminants du Massif central à l'horizon 2050. In : Élevage bovin allaitant. Agabriel J., Renand G., Baumont R. (Eds). Dossier, INRA Prod. Anim., 30, 179-196. 
